Swarthmore College

Works

$11-1-2011$

\title{
Three-Dimensional Magnetohydrodynamics Simulations Of Counter-Helicity Spheromak Merging In The Swarthmore Spheromak Experiment
}

\author{
C. E. Myers \\ E. V. Belova \\ Michael R. Brown \\ Swarthmore College,doc@swarthmore.edu \\ Timothy G. Gray , '01 \\ C. D. Cothran
}

See next page for additional authors

Follow this and additional works at: https://works.swarthmore.edu/fac-physics

Part of the Physics Commons

Let us know how access to these works benefits you

\section{Recommended Citation}

C. E. Myers; E. V. Belova; Michael R. Brown; Timothy G. Gray , '01; C. D. Cothran; and M. J. Schaffer. (2011). "Three-Dimensional Magnetohydrodynamics Simulations Of Counter-Helicity Spheromak Merging In The Swarthmore Spheromak Experiment". Physics Of Plasmas. Volume 18, Issue 11. DOI: 10.1063/1.3660533 https://works.swarthmore.edu/fac-physics/106

This work is brought to you for free by Swarthmore College Libraries' Works. It has been accepted for inclusion in Physics \& Astronomy Faculty Works by an authorized administrator of Works. For more information, please contact myworks@swarthmore.edu. 


\section{Authors}

C. E. Myers; E. V. Belova; Michael R. Brown; Timothy G. Gray , '01; C. D. Cothran; and M. J. Schaffer 


\section{AIP $\left.\right|_{\text {Physics of }}$ \\ Plasmas}

\section{Three-dimensional magnetohydrodynamics simulations of counter-helicity spheromak merging in the Swarthmore Spheromak Experiment}

C. E. Myers, E. V. Belova, M. R. Brown, T. Gray, C. D. Cothran, and M. J. Schaffer

Citation: Physics of Plasmas (1994-present) 18, 112512 (2011); doi: 10.1063/1.3660533

View online: http://dx.doi.org/10.1063/1.3660533

View Table of Contents: http://scitation.aip.org/content/aip/journal/pop/18/11?ver=pdfcov

Published by the AIP Publishing

Articles you may be interested in

Nonlinear electron-magnetohydrodynamic simulations of three dimensional current shear instability

Phys. Plasmas 19, 092305 (2012); 10.1063/1.4751872

Nonlinear three-dimensional verification of the SPECYL and PIXIE3D magnetohydrodynamics codes for fusion plasmas

Phys. Plasmas 17, 082501 (2010); 10.1063/1.3462908

The scaling properties of dissipation in incompressible isotropic three-dimensional magnetohydrodynamic turbulence

Phys. Plasmas 12, 022301 (2005); 10.1063/1.1842133

The three-dimensional magnetohydrodynamics of ac helicity injection in the reversed field pinch Phys. Plasmas 10, 999 (2003); 10.1063/1.1555622

Magnetohydrodynamic simulation on co- and counter-helicity merging of spheromaks and driven magnetic reconnection

Phys. Plasmas 4, 1297 (1997); 10.1063/1.872305

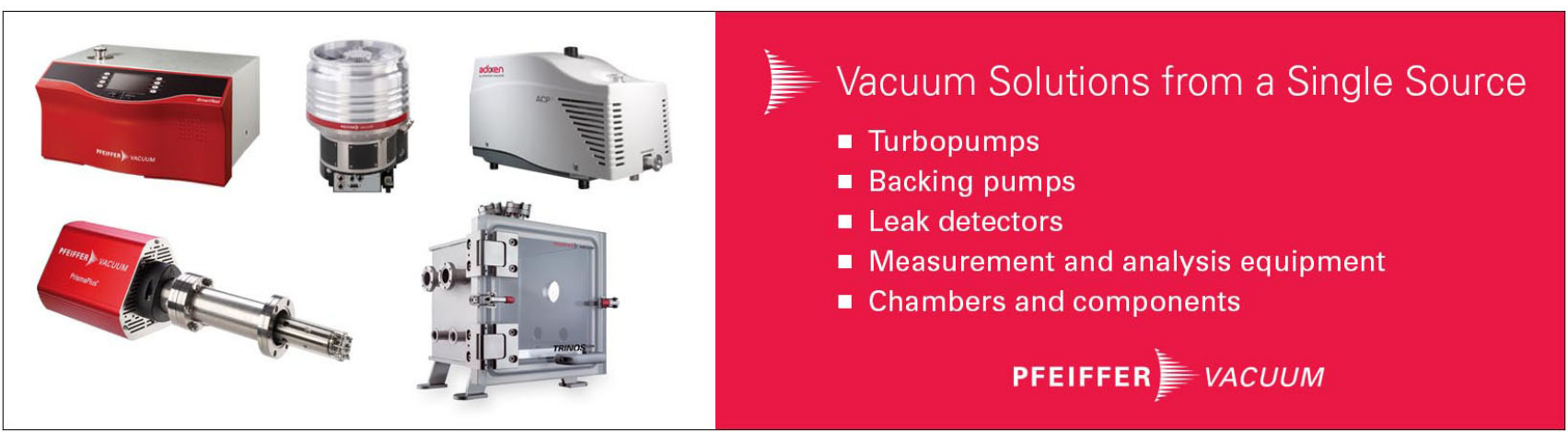




\title{
Three-dimensional magnetohydrodynamics simulations of counter-helicity spheromak merging in the Swarthmore Spheromak Experiment
}

\author{
C. E. Myers, ${ }^{1, a)}$ E. V. Belova, ${ }^{1}$ M. R. Brown, ${ }^{2}$ T. Gray, ${ }^{2}$ C. D. Cothran, ${ }^{2, b)}$ and M. J. Schaffer ${ }^{3}$ \\ ${ }^{1}$ Princeton Plasma Physics Laboratory, Princeton, New Jersey 08543, USA \\ ${ }^{2}$ Department of Physics and Astronomy, Swarthmore College, Swarthmore, Pennsylvania 19081, USA \\ ${ }^{3}$ General Atomics, San Diego, California 92186, USA
}

(Received 9 September 2011; accepted 16 October 2011; published online 30 November 2011)

\begin{abstract}
Recent counter-helicity spheromak merging experiments in the Swarthmore Spheromak Experiment (SSX) have produced a novel compact torus (CT) with unusual features. These include a persistent antisymmetric toroidal magnetic field profile and a slow, nonlinear emergence of the $n=1$ tilt mode. Experimental measurements are inconclusive as to whether this unique CT is a fully merged field-reversed configuration (FRC) with strong toroidal field or a partially merged "doublet CT" configuration with both spheromak- and FRC-like characteristics. In this paper, the SSX merging process is studied in detail using three-dimensional resistive MHD simulations from the Hybrid Magnetohydrodynamics (HYM) code. These simulations show that merging plasmas in the SSX parameter regime only partially reconnect, leaving behind a doublet CT rather than an FRC. Through direct comparisons, we show that the magnetic structure in the simulations is highly consistent with the SSX experimental observations. We also find that the $n=1$ tilt mode begins as a fast growing linear mode that evolves into a slower-growing nonlinear mode before being detected experimentally. A simulation parameter scan over resistivity, viscosity, and line-tying shows that these parameters can strongly affect the behavior of both the merging process and the tilt mode. In fact, merging in certain parameter regimes is found to produce a toroidal-field-free FRC rather than a doublet CT. (C) 2011 American Institute of Physics. [doi:10.1063/1.3660533]
\end{abstract}

\section{INTRODUCTION}

Spheromaks ${ }^{1,2}$ and field-reversed configurations ${ }^{3-6}$ (FRCs) are compact, simply connected magnetic fusion plasmas. Fusion reactor designs that are based on these compact torus (CT) plasmas are much simpler to develop and build than standard tokamak-based reactor designs. FRC-based reactors in particular are attractive because of their natural divertor geometry and large $\beta$ limit. The large $\beta$ limit reduces the external magnetic field requirements for the reactor and accommodates the use of cleaner aneutronic fusion fuels.

In order to realize the potential of FRC-based fusion reactors, however, the performance of experimental FRC plasmas must be significantly improved. Two key areas for improvement are (1) formation and (2) stability. With regard to formation, much of the experimental success to date has been achieved with the field-reversed $\theta$-pinch (FRTP) formation process. ${ }^{3}$ FRTP-formed FRCs are prolate (elongated) and highly kinetic with $S^{*} \lesssim 10$. Here, the kinetic parameter $S^{*}$ is defined as $S^{*} \equiv R_{s} / \lambda_{i} \simeq R_{S} / \rho_{i, \text { ext }}$, where $R_{s}$ is the FRC's separatrix radius, $\lambda_{i} \equiv c / \omega_{p i}$ is the ion skin depth, and $\rho_{i, \text { ext }}$ is the ion gyroradius in the external field. Unfortunately, FRTP formation requires high-voltage pulsed power supplies that do not scale favorably to reactor size. Additionally, a reactor-grade FRC will likely operate in a more MHD-like regime with $S^{*} \gtrsim 40$. Thus, in order to progress toward a feasible FRC-based fusion reactor, a scalable (low-

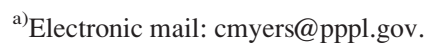

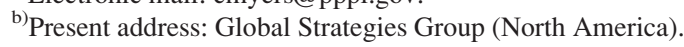

voltage) formation technique that can produce MHD-like FRCs must be developed. One candidate is the rotating magnetic field (RMF) technique. Here, a multi-component antenna set is used to rotate a perturbative magnetic field through the plasma. In recent years, RMF devices have produced prolate FRCs with $T_{e} \simeq 150-200 \mathrm{eV}$. $^{7,8}$ Another promising low-voltage FRC formation technique, counterhelicity spheromak merging, produces high-flux oblate FRCs and is the subject of this paper.

With regard to stability, both spheromaks and FRCs are theoretically unstable to an ideal MHD $n=1$ tilt mode. ${ }^{9}$ Experimentally, FRCs produced by FRTP formation have been observed to be relatively stable to this tilt mode. This is likely due to finite Larmor radius (FLR) effects and nonlinear stabilization; ${ }^{10,11}$ however, some evidence of tilting has been observed even in highly kinetic FRCs. ${ }^{12}$ Spheromaks, which are inherently more MHD-like, are typically stabilized against the tilt mode using a combination of equilibrium field coils and a close-fitting flux conserver. ${ }^{9,13,14}$ It has been shown that a cylindrical flux conserver is stabilizing if it is oblate with a length-to-radius ratio $L / R \leq 1.67 .^{15-17}$ In order to improve the performance of fusion-relevant CT plasmas, we must continue to develop our understanding of instabilities such as the tilt mode.

The SSX-FRC device is designed to study advanced formation and stabilization techniques that will hopefully lead to higher-performance FRC plasmas. Formation in SSX-FRC is accomplished using the aforementioned counter-helicity spheromak merging technique. ${ }^{18}$ Originally developed by Yamada and Ono in the TS-3 device, ${ }^{19}$ counter-helicity merging is 
initiated by generating two side-by-side spheromaks of opposite helicity. The parallel currents in the two spheromaks draw them together and drive them to reconnect. Ideally, this process annihilates the oppositely directed toroidal field in the two spheromaks and leaves behind a toroidal-field-free FRC plasma. Also, because poloidal flux is amplified during relaxation, ${ }^{13}$ a large amount of poloidal flux is entrained in the resulting FRC.

Stabilization in SSX-FRC is provided by a quasicylindrical copper flux conserver. Several different flux conservers with various shapes and elongations have been implemented in SSX, but no central conductor (center stack) has ever been used. Thus, all SSX plasmas are true simply connected compact toroids. In this paper, we focus on counter-helicity merging experiments that were conducted in a prolate $(L / R=3)$ cylindrical flux conserver. This flux conserver, which is (intentionally) tilt-unstable, is designed to study the development of the tilt instability in various CT plasmas.

The primary goal of these prolate SSX-FRC experiments was to produce and study fully merged FRC plasmas. During the experimental campaign, however, prototypical FRC plasmas with only poloidal field were never observed. Instead, a novel CT with unusual features is formed. ${ }^{18}$ Experimentally, this CT is characterized by a strong antisymmetric toroidal field profile and a slow, nonlinear emergence of the $n=1$ tilt instability. These results are surprising given the successful formation of toroidal-field-free FRCs via counter-helicity merging in the TS-3 device. ${ }^{20}$ Consequently, understanding why spheromak merging is inherently different in SSX is of great interest to recent experimental campaigns in devices such as TS-3/4, ${ }^{21}$ the Magnetic Reconnection Experiment $(\mathrm{MRX})^{22}$ and the C-2 device at Tri-Alpha Energy. ${ }^{23}$ In C-2, two FRTP-formed FRCs are supersonically merged to form a single flux-amplified FRC.

Several recent numerical simulation results may explain the SSX-FRC observations. On one hand, hybrid simulations by Omelchenko et al. ${ }^{24,25}$ with the FLAME code have shown that an antisymmetric toroidal field profile can develop in large- $S^{*}$ FRCs. It is found that the presence of this toroidal field has a stabilizing influence on the tilt mode. Alternatively, resistive MHD simulations by Belova et al. ${ }^{26}$ with the Hybrid Magnetohydrodynamics (HYM) $\operatorname{code}^{27}$ have shown that large ion viscosity in the plasma can slow both the merging process and the growth of the tilt mode. The stalled merging process leaves behind a partially merged configuration with two sets of spheromak-like flux surfaces embedded in a single set of FRC-like flux surfaces. In this "doublet CT" configuration, the measured toroidal field profile is simply a remnant of the initial toroidal field that is carried in by the two merging spheromaks.

Despite these simulation efforts, it remains unclear which of these scenarios accounts for the merging behavior that is observed in SSX-FRC. In this paper, we resolve this debate by showing that nearly all of the salient features of the SSXFRC merging process are consistent with the formation of a partially merged, tilt-unstable doublet CT. This conclusion is drawn from direct comparisons between the SSX-FRC experimental data and new three-dimensional resistive MHD simulations from the HYM code. These simulations are designed to be as realistic as possible and their evolution is highly consistent with the experimental data. This work also includes an exploration of the role of key plasma parameters including resistivity, viscosity, and line-tying. Thus, not only do we establish what happens in the SSX-FRC merging process, we also identify the most important physical mechanisms that drive this behavior.

The paper is organized as follows: In Sec. II, we motivate the present work by describing the relevant SSX-FRC experimental results. Then, in Sec. III, we detail the computational framework that has been implemented in the HYM code to accurately simulate the SSX-FRC merging process. Section IV contains the simulation results for SSX-like plasmas, including direct comparisons to the experimental data. Detailed analysis of MHD phenomena in the reconnection region is reported in Sec. V and Sec. VI presents a multidimensional scan of three important plasma parameters (resistivity, viscosity, and line-tying). This parameter scan, which helps to place the simulation results in a broader context, reveals that the merging behavior can be altered by changes in the plasma parameters.

\section{MOTIVATION: SSX-FRC EXPERIMENTS}

In this section, we describe the SSX-FRC device and the experimental results that motivate the simulation work presented in this paper. The magnetic diagnostics used in SSXFRC are emphasized because data from these diagnostics will later be directly compared to data from the HYM simulations. Results from additional SSX-FRC diagnostics are discussed in the appendix.

\section{A. The SSX-FRC device}

The SSX-FRC device in its prolate configuration is described elsewhere ${ }^{18}$ so we only briefly review it here. In SSX-FRC, opposing coaxial magnetized plasma guns produce spheromaks of either helicity at either end of the device (see Fig. 1). Each plasma gun is surrounded by a "stuffing flux" coil that seeds the poloidal field for each spheromak during formation. The guns are typically operated at $\sim 1$ $\mathrm{mWb}$ of magnetic flux, but the poloidal flux in each spheromak is amplified to $\sim 3-4 \mathrm{mWb}$ during relaxation. In the counter-helicity merging configuration, the gun flux diverges from one gun and converges back to the other, producing a mirror-like background field and spheromaks with parallel toroidal currents.

Spheromak merging is unique in SSX because fully ionized spheromaks are formed remotely and injected into a high vacuum, low magnetic field merging zone with no solid central column. This merging zone is bounded by a quasicylindrical copper flux conserver that is suspended inside the vacuum vessel. Several different flux conservers have been used in SSX with radii ranging from $R=8.5-25.0 \mathrm{~cm}$. The prolate flux conserver that was used in nearly all of the experiments discussed here has $R=20.3 \mathrm{~cm}$ and $L=61.0$ $\mathrm{cm}$ (a length-to-radius ratio of $L / R=3$, which is deliberately tilt unstable).

The primary diagnostic that is used to characterize the spheromak merging process in SSX-FRC is an array of 


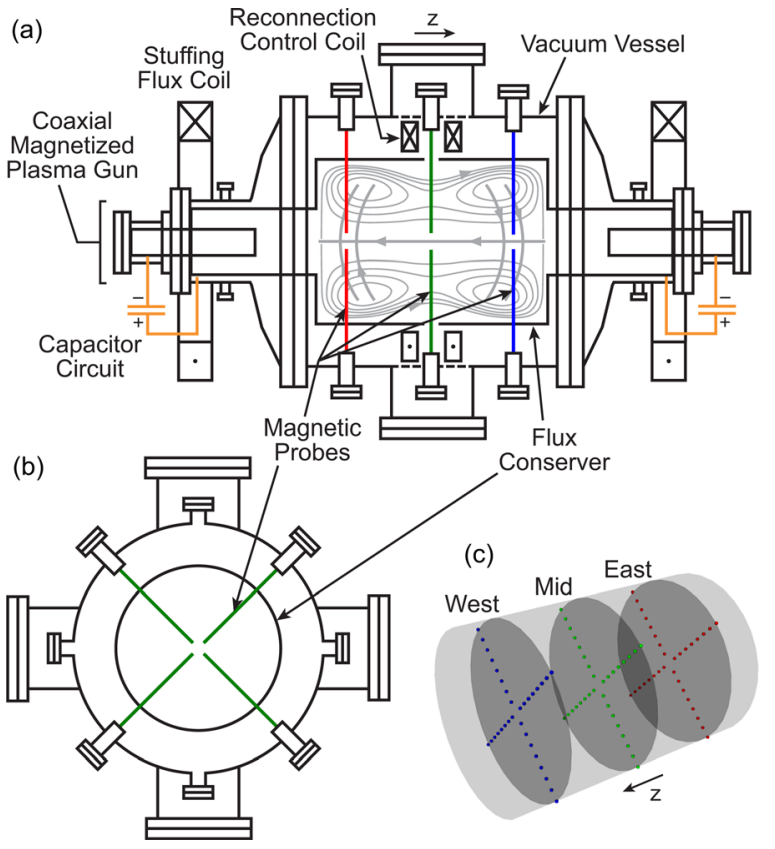

FIG. 1. (Color online) SSX in its prolate SSX-FRC configuration. (a) A $z-r$ cross-section of the experiment showing the locations of the magnetic probes. A set of a set of calculated poloidal flux contours for $B_{R C C}=210 \mathrm{G}$ is shown in gray. (b) A corresponding $r-\theta$ cross-section of the experiment. (c) A 3D rendering of the 96 magnetic field measurement locations.

twelve linear magnetic probes (see Fig. 1). Each probe measures all three components of the magnetic field $\left(B_{z}, B_{r}, B_{\theta}\right)$ at eight radial locations along its length for a total of $12 \times 8=96$ measurement locations inside the plasma. The radial spacing between the measurement locations is $2.5 \mathrm{~cm}$. The twelve linear probes are distributed across three axial locations within the flux conserver: one at the midplane $(z=0 \mathrm{~cm})$ and the others at the "west" and "east" locations $(z= \pm 21.6 \mathrm{~cm})$. At each axial location, four probes are inserted at equally spaced toroidal locations to permit toroidal mode decomposition $(n=0,1)$. The measurements from the magnetic probes are digitized at $0.8 \mu$ s intervals and can thus be used to examine both the temporal and spatial evolution of the plasma.

\section{B. Experimental results}

The counter-helicity merging process in SSX-FRC is mediated by two "Reconnection Control Coils" (RCCs) that are located near the midplane of the device. Calculations from the finite element code EQLFE (Ref. 28) show that the character of the expected post-merging equilibrium depends on the strength of the applied RCC field, $B_{R C C}$. In particular, modest-to-high RCC values $\left(B_{R C C} \gtrsim 140 \mathrm{G}\right)$ should produce a partially merged "doublet CT" with two distinct regions of private flux surrounded by a region of shared flux (see Fig. 1(a)); low RCC values $\left(B_{R C C} \lesssim 70 \mathrm{G}\right)$ on the other hand are expected to produce a fully merged FRC. As shown in Cothran et al., ${ }^{18}$ the amount of reconnected (shared) flux at the midplane is reduced (as expected) by high values of $B_{R C C}$. At low values of $B_{R C C}$, however, the expected fully merged FRC equilibrium is not achieved.

To illustrate this behavior, we present the magnetics data from a typical low- $B_{R C C}$ discharge $\left(B_{R C C}=70 \mathrm{G}\right)$. First, we examine the time evolution of the distribution of magnetic energy in the plasma. The toroidal arrangement of the magnetic probes (see Fig. 1(c)) permits the Fourier decomposition of the measured magnetic field components $\left(B_{z}, B_{r}\right.$, $B_{\theta}$ ) into their constituent $n=0,1$ toroidal mode amplitudes. These mode amplitudes are converted to poloidal and toroidal mode energies, $w_{m, p o l}^{n}$ and $w_{m, t o r}^{n}$, before being radially averaged to reduce the data to just four waveforms $(n=0,1 \times$ pol,tor $)$ at each of the three axial probe positions. This analysis procedure, which is more thoroughly explained in Cothran et al. ${ }^{18}$ and in Sec. IV B of this paper, produces a total of twelve radially averaged mode energy density waveforms, $\left\langle w_{m, p o l}^{n}\right\rangle$ and $\left\langle w_{m, t o r}^{n}\right\rangle$.

The mode energy waveforms from a typical SSX-FRC counter-helicity discharge are shown in Fig. 2. The "injection" phase of the discharge (Phase I) begins at $t=0 \mu$ s with the firing of the capacitor banks. At this time, the neutral gas in the plasma guns breaks down and the spheromaks begin to form. Strong $\mathbf{J} \times \mathbf{B}$ forces within the guns drive the spheromaks out into the flux conserver. Phase I lasts until $t \simeq 25 \mu$ s when the off-midplane probes begin to measure the $n=0$ poloidal and toroidal magnetic energy that is carried toward the midplane by the two injected spheromaks.

The axisymmetric "merging" phase of the discharge (Phase II), which lasts from $t \simeq 25-80 \mu \mathrm{s}$, begins with the detection of large $n=0$ energy (both poloidal and toroidal) at the off-midplane probe locations. This measured energy indicates that the two newly formed spheromaks have entered the flux conserver. When the spheromaks collide shortly thereafter, $n=0$ poloidal magnetic energy begins to accumulate at the midplane. This behavior indicates that pairs of flux surfaces from the two spheromaks are reconnecting to form shared flux surfaces that straddle the midplane. In prototypical counter-helicity merging, this process continues until all of the private flux surfaces have reconnected and the axisymmetric toroidal field is completely

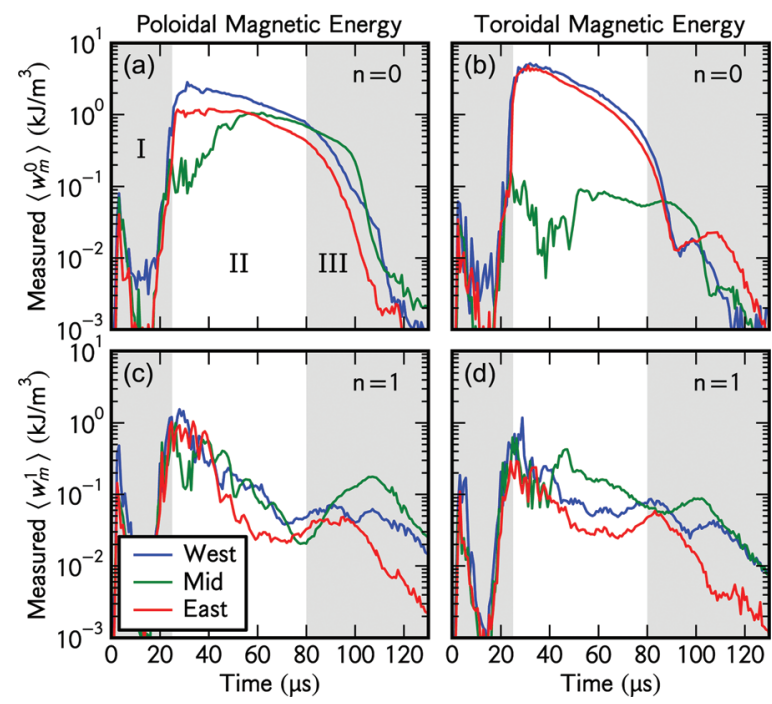

FIG. 2. (Color) Time evolution of the measured $n=0$ and $n=1$ poloidal and toroidal magnetic energies in a typical counter-helicity discharge in SSX-FRC. In each plot, signals are shown from both the midplane and the off-midplane (east and west) probe locations. The discharge is demarcated into three phases: (I) injection; (II) merging; and (III) tilt. 
annihilated. In these SSX-FRC discharges, however, significant $n=0$ toroidal field is detected by the off-midplane probes even after the growth of the midplane poloidal energy (and hence the reconnection process) has stagnated.

Phase III of the SSX-FRC discharge, the "tilt" phase, begins at $t \simeq 80 \mu \mathrm{s}$ and is dominated by a sharp decline in both the poloidal and toroidal $n=0$ mode energies. This decline is driven by the emergence of the $n=1$ tilt mode, which is evident between $t \simeq 80-100 \mu$ s with the rise in the measured $n=1$ energy densities (see, in particular, the midplane waveform in Fig. 2(c)). The damped $n=1$ energy measured prior to this time is attributed to inherent nonaxisymmetries introduced during the spheromak formation process. Note that additional probes were added to the offmidplane measurement locations in order to resolve the $n=2,3$ modes, but that the measured amplitudes of these higher- $n$ modes were found to be insignificant compared to those of the $n=0,1$ modes. It is interesting to note that the $n=1$ tilt mode takes more than 15 radial Alfvén times (5 axial Alfvén times) to emerge macroscopically. Here, the radial Alfvén time $t_{A 0} \simeq 3 \mu \mathrm{s}$ is defined as $t_{A 0} \equiv R_{C} / v_{A 0}$, where $R_{c}=20.3 \mathrm{~cm}$ is the radius of the flux conserver and $v_{A 0} \simeq 70 \mathrm{~km} / \mathrm{s}$ is the typical Alfvén velocity in SSX-FRC. When the tilt mode is finally detected, it has a characteristic growth time of $\gamma_{1}^{-1} \sim 6-8 t_{A 0}$, which is much slower than the expected linear growth time of $\gamma_{1}^{-1} \simeq t_{A 0}$.

In addition to the mode energy waveforms shown above, it is also useful to examine single-time snapshots of the magnetic structure of the plasma. One such snapshot (at $t=62.4$ $\mu \mathrm{s})$ is shown in Fig. 3. Vector plots of the raw magnetic data are shown in (a), while plots of the filtered $n=0$ components are shown in (b). The $n=0$ vector plots reveal the salient features of the merged plasma. In particular, the residual antisymmetric toroidal field is evident from the off-midplane $r-\theta$ plots, while the general poloidal field structure is visible in the two $z-r$ plots. Unfortunately, the sparse distribution of magnetic measurements in the axial direction does not reveal whether the merged plasma has private flux regions on either side of the midplane. These interesting and unexplained features of the SSX-FRC merging process motivate the simulation work presented in this paper.

\section{THE HYM-SSX COMPUTATIONAL FRAMEWORK}

The HYM code is a fully parallelized, Fortran-90-based code that is capable of simulating a variety of fusionrelevant plasmas. HYM is a fourth order finite difference code with an explicit second order centered-in-time stepping scheme. Message Passing Interface (MPI) is used to facilitate domain decomposition in up to three dimensions. Simulations can be performed in any orthogonal coordinate system and the code is written with a modular structure that permits the simulation of a wide range of configurations. Accordingly, the code has been adapted to run in many different experimental geometries including that of the SSX-FRC $L / R=3$ flux conserver introduced here.

Fundamentally, HYM is a hybrid code that can implement various combinations of the MHD, two-fluid, and kinetic plasma models. For the simulations in this paper, however, (a) Magnetic vectors (raw)

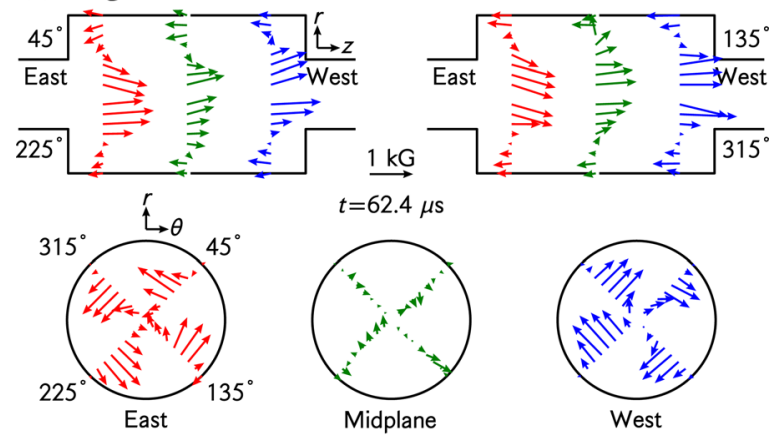

(b) Magnetic vectors $(\mathrm{n}=0)$

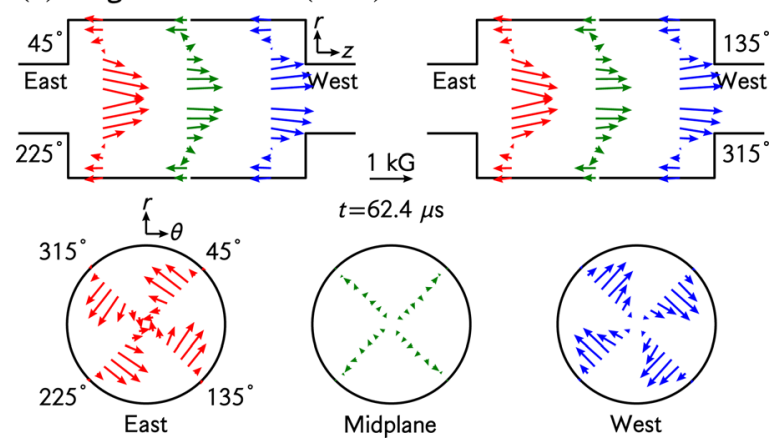

FIG. 3. (Color online) 2D projections of the SSX-FRC magnetics measurements at $t=62.4 \mu \mathrm{s}$ : (a) the raw vector magnetics data and (b) the $n=0$ component showing the prevailing axisymmetric structure of the plasma at this time.

only the resistive MHD model is used. The validity of the resistive MHD model in the SSX parameter regime has been verified with an additional set of two fluid simulations (see Sec. IV C). The simulations presented here were run on the Franklin computer at the National Energy Research Scientific Computing Center (NERSC) using up to 1000 parallelized cores. For production runs, meshes with $513 \times 129 \times 32$ $\left(N_{z} \times N_{r} \times N_{\theta}\right)$ nodes are used. High resolution runs such as these consume approximately 3000 processor-hours each.

In its MHD-only operational mode, the HYM code solves the nonlinear resistive MHD equations with finite scalar resistivity and viscosity

$$
\begin{gathered}
\frac{\partial \rho}{\partial t}+\nabla \cdot(\rho \mathbf{v})=0 \\
\frac{\partial \rho \mathbf{v}}{\partial t}+\nabla \cdot(\rho \mathbf{v v})=\mathbf{J} \times \mathbf{B}-\nabla p+\mu \nabla^{2} \mathbf{v} \\
\frac{\partial p}{\partial t}+\mathbf{v} \cdot \nabla p+\gamma p(\nabla \cdot \mathbf{v})=(\gamma-1) \\
\times\left[\alpha_{O H}\left(\eta J^{2}\right)+\alpha_{V H}\left(\mu(\nabla \times \mathbf{v})^{2}+\mu(\nabla \cdot \mathbf{v})^{2}\right)\right], \\
\mathbf{E}+\mathbf{v} \times \mathbf{B}=\eta \mathbf{J} \\
\mathbf{B}=\nabla \times \mathbf{A} \\
\nabla \times \nabla \times \mathbf{A}=\mu_{0} \mathbf{J} \\
\frac{\partial \mathbf{A}}{\partial t}=-\mathbf{E} .
\end{gathered}
$$

In these dimensionless equations, $\rho$ is the mass density, $\mathbf{v}$ is the fluid velocity, $\mathbf{J}$ is the current density, $\mathbf{B}$ is the magnetic 
field, $p$ is the plasma pressure, $\mathbf{E}$ is the electric field, and $\mathbf{A}$ is the magnetic vector potential. The scalar resistivity $\eta$ is parameterized in the code as $R_{c} / S$, where $S$ is the Lundquist number and $R_{c}$ is the typical MHD scale length of the plasma in units of the ion skin depth $\lambda_{i} \equiv c / \omega_{p i}$. For these simulations, the radius of the SSX flux conserver, $R_{c}=20.3 \mathrm{~cm} \simeq 28.2 \lambda_{i}$, is used. In the same way, the scalar viscosity $\mu$ is parameterized as $R_{c} / \mathrm{Re}$, where $\mathrm{Re}$ is the fluid Reynolds number. Both $\eta$ and $\mu$ are prescribed constants in time and space.

The pressure evolution equation used in the resistive MHD version of HYM (Eq. (3)) is formulated so as to conserve energy throughout the simulation. In particular, the terms on the right hand side of Eq. (3) represent Ohmic heating $(\mathrm{OH})$ and viscous heating $(\mathrm{VH})$, respectively. Terms that account for loss mechanisms such as heat conduction and radiative transport are neglected. In order to partially compensate for these neglected loss terms, the constants $\alpha_{\mathrm{OH}}$ and $\alpha_{V H}$ are introduced into Eq. (3). Setting either of these constants to a value less than unity inhibits the corresponding heating term, which, in turn, crudely accounts for some of the neglected loss mechanisms. For the simulations presented in this paper, $\alpha_{O H}=0.5$ and $0.0 \leq \alpha_{V H} \leq 1.0$. It was found here that the precise value of $\alpha_{V H}$ is unimportant in these simulations because the $\mathrm{OH}$ term tends to dominate even for $\alpha_{V H}=1.0$.

\section{A. HYM-SSX boundary conditions and initial conditions}

An important part of accurately simulating the SSXFRC merging process is to set up realistic boundary conditions and initial conditions for the simulations. Accordingly, the boundary of the HYM-SSX simulation domain is defined by a cylinder with the dimensions of the $L / R=3$ flux conserver in SSX-FRC. This cylindrical boundary is perfectly conducting, which is appropriate given the long skin time of the SSX-FRC copper flux conserver $(>1 \mathrm{~ms})$ as compared to the plasma discharge time $(\sim 100 \mu \mathrm{s})$. Contributions from several external magnetic field coils (e.g., the stuffing flux coils and the reconnection control coils) are frozen into the flux conserver boundary for the duration of the simulation.

Due to the simulation boundary conditions, the injection of the spheromaks by the coaxial magnetized guns through the ends of the flux conserver is not simulated. Instead, the initial conditions for the simulations are designed to approximate the state of the spheromaks shortly after they exit the plasma guns and relax inside the ends of the flux conserver. To do this, we use a quasi-equilibrium approach to calculating the initial conditions. We first solve the Grad-Shafranov (GS) equation for a spheromak equilibrium in half of the SSX-FRC flux conserver. The resulting solution is then reflected across the midplane so that a second spheromak is placed in the other half of the domain. This reflection preserves the direction of the toroidal current in the second spheromak but reverses its toroidal field so that initial conditions with two counter-helicity spheromaks are produced. Note that the two side-by-side spheromaks are not in true equilibrium because their parallel toroidal currents will pull them together when the simulation begins.
The HYM-SSX initial conditions are composed of axisymmetric 2D $(z-r)$ spatial profiles of several quantities including the poloidal flux $\psi_{p}$, toroidal field $B_{\text {tor }}$, pressure $p$, mass density $\rho$, and current density J. Sample initial poloidal flux and toroidal field profiles are displayed in Figs. 4(a) and 4(b), respectively. This arrangement of poloidal flux and toroidal field gives rise to a monotonic $q\left(\psi_{p}\right)$ profile within each spheromak (see Fig. 4(c)). In this setup, the last closed flux surface (LCFS) is limited by the end cap of the flux conserver.

A potentially important experimental effect is that of axial line-tying from field lines that stream back into the plasma guns. Line-tying is incorporated in these simulations by first solving for the quasi-equilibrium GS solution in a flux conserver that is slightly longer than the SSX-FRC flux conserver. When this modified GS solution is placed back inside the original flux conserver, the last few flux surfaces of each spheromak are cut off and tied to the perfectly conducting end caps. The relative amount of line-tying that is added using this technique is quantified by the axial expansion factor $\zeta_{L T} \equiv 2 \Delta z / L_{c}$, where $L_{c} / 2$ is half of the length of the original flux conserver and $\Delta z$ is width of the axial expansion at each end of the domain. Sample line-tied flux profiles $\left(\psi_{p, L T}(r)\right.$ at $\left.z= \pm L_{c} / 2\right)$ are displayed in Fig. 4(d) for several values of $\zeta_{L T}$. Note that the line-tied flux is only a small fraction of the peak flux found at the center of each spheromak.

The final component of the HYM-SSX initial conditions is a $3 \mathrm{D}$ perturbation to the initial vector fluid velocity profile. Note that all of the initial conditions described thus far have been $2 \mathrm{D}$, so it is this initial velocity perturbation that introduces 3D behavior into the simulations. For the simulations described in this paper, this initial velocity perturbation is
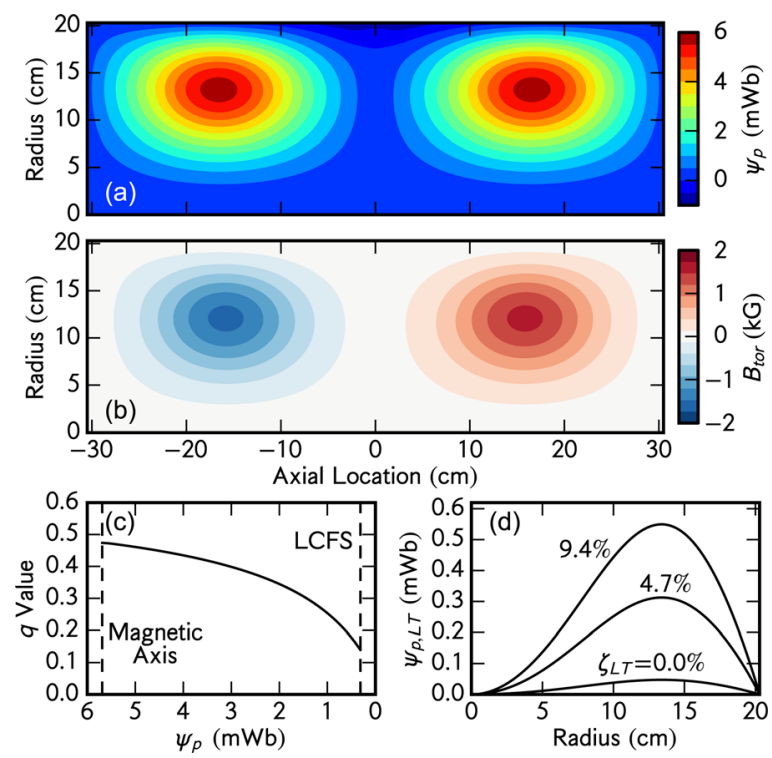

FIG. 4. (Color online) Sample initial conditions for the HYM-SSX simulations: (a) The 2D (z-r) spatial profile of the poloidal flux $\psi_{p}$. (b) The 2D profile of the toroidal field $B_{t o r}$. (c) The $q\left(\psi_{p}\right)$ profile moving outward from the magnetic axis of one of the spheromaks to its LCFS. (d) Radial line-tied flux profiles $\psi_{p, L T}(r)$ at the end caps of the flux conserver $\left(z= \pm L_{c} / 2\right)$ for several values of the axial expansion factor $\zeta_{L T}$. Note that this "line-tying parameter" is $\zeta_{L T}=4.7 \%$ for the sample initial conditions shown in (a)-(c). 
turbulent (i.e., randomized at each location). It is also scaled spatially with the magnitude of the poloidal flux. Note that this type of perturbation does not drive the two spheromaks toward the midplane; instead they are simply left to accelerate toward each other as a result of the attractive force between their parallel currents.

\section{B. HYM-SSX simulation parameters}

A primary goal of this work is to accurately simulate the merging process in SSX-FRC. In order to do this, the available set of simulation parameters must be chosen to be as close as possible to the known plasma conditions in SSXFRC. The key parameter values that were used for these simulations are displayed in Table I. Many of these parameters such as $B_{0}$ are well established by measurements from the experiments. Additional experimental measurements that help to constrain parameters such as $n_{0}$ and $T_{\text {tot }}=T_{i}+T_{e}$ are detailed in the appendix.

Other simulation parameters are not so easily constrained. These include the resistivity $\eta$, the viscosity $\mu$, and the line-tying parameter $\zeta_{L T}$. From a classical resistivity estimate, $S \simeq 500-1000$ for SSX-FRC, which agrees with the experimentally observed plasma decay rate. Thus, the simulation value of $S=700$ is in the proper regime. Obtaining an accurate estimate of the viscosity is more challenging. There are several estimates that could be employed. Using $T_{i} \sim 12$ $\mathrm{eV}$ and the other relevant parameters from Table I, the Braginskii unmagnetized ion viscosity gives an estimate of $\operatorname{Re} \simeq 120$, while the Braginskii weakly magnetized ion viscosity gives $\mathrm{Re} \simeq 600$. Finally, the Braginskii gyro viscosity gives $\operatorname{Re} \simeq 200$. This wide range of estimates gives little indication as to the appropriate value of Re. The simulation value of $\mathrm{Re}=700$ was chosen based on various detailed comparisons between the simulations and the SSX-FRC measurements. The same is true for the line-tying parameter $\zeta_{L T}$, which at the value of $\zeta_{L T}=4.7 \%$ has a modest effect on the simulations (see Sec. VI).

\section{SIMULATIONS OF SSX-LIKE PLASMAS}

\section{A. Simulation results}

In this section, we present results from HYM simulations of merging plasmas in the SSX parameter regime.

TABLE I. Parameters for the HYM-SSX simulations.

\begin{tabular}{lc}
\hline \hline Magnetic Field (Edge) & $B_{0}=1.0 \mathrm{kG}$ \\
Density (Peak) & $n_{0}=10^{15} \mathrm{~cm}^{-3}$ \\
Nominal Temperature & $T_{t o t}=T_{i}+T_{e} \simeq 25 \mathrm{eV}$ \\
Ion Skin Depth & $\lambda_{i}=c / \omega_{p i} \simeq 0.72 \mathrm{~cm}$ \\
MHD Scale Length (Radial) & $R_{c}=20.3 \mathrm{~cm} \simeq 28.2 \lambda_{i}$ \\
Alfvén Velocity & $v_{A 0}=69.0 \mathrm{~km} / \mathrm{s}$ \\
Alfvén Time (Radial) & $t_{A 0}=R_{C} / v_{A 0} \simeq 2.94 \mu \mathrm{s}$ \\
Adiabatic Index & $\gamma=5 / 3$ \\
Resistivity & $\eta=0.040(S \simeq 700)$ \\
Viscosity & $\mu=0.040(\mathrm{Re} \simeq 700)$ \\
Applied RCC Field & $B_{R C C}=70 \mathrm{G}$ \\
Line-Tying Parameter & $\zeta_{L T}=4.7 \%$ \\
Init. Velocity Perturbation & $v_{0, \max }=0.05 v_{A 0}$ \\
\hline \hline
\end{tabular}

Because the initial conditions described in Sec. III A roughly correspond to the end of the spheromak injection process, the simulations begin at the start of Phase II of the discharge ( $t \simeq 25 \mu \mathrm{s}$ ). Over the next $\sim 45 \mu \mathrm{s}$, flux surfaces from the two counter-helicity spheromaks proceed to reconnect. Before the merging process can complete, however, the reconnection process stagnates. The resulting configuration is a doublet CT with regions of both public and private flux (see Fig. 5(a)). The two private flux regions retain most of the antisymmetric toroidal field that is initially carried in by the two merging spheromaks (see Fig. 5(b)).

To quantify the degree to which the two spheromaks merge, we introduce a simulation metric that we call the merging fraction $R_{\text {merg }}$

$$
R_{\text {merg }}(t) \equiv 1-\left(\frac{z_{+}(t)-z_{-}(t)}{z_{+0}-z_{-0}}\right)^{2},
$$

where $z_{+}\left(z_{-}\right)$is the axial location of the poloidal flux peak in the right (left) half of the flux conserver. At the beginning of the simulation, $R_{\text {merg }}=0 \%$ because $z_{+}=z_{+0}$ and $z_{-}=z_{-0}$. If, on the other hand, the spheromaks fully merge at some later time, then $z_{+}=z_{-}$and $R_{\text {merg }}=100 \%$. This metric is very useful for comparing the relative behavior of simulations with different plasma parameters. For SSX-like plasmas, $R_{\text {merg }}$ climbs steadily until $t \simeq 70 \mu$ s when it saturates at $R_{\text {merg }} \simeq 50 \%$ (see Fig. 5(c)). During this time, the total $n=0$ toroidal magnetic energy, $W_{m \text {.tor }}^{0}$, slowly declines due to both reconnection and resistive decay (see Fig. 5(d)). Because the merging process remains incomplete, however, significant toroidal magnetic energy persists until the $n=1$ tilt mode emerges around $t \simeq 80 \mu \mathrm{s}$.
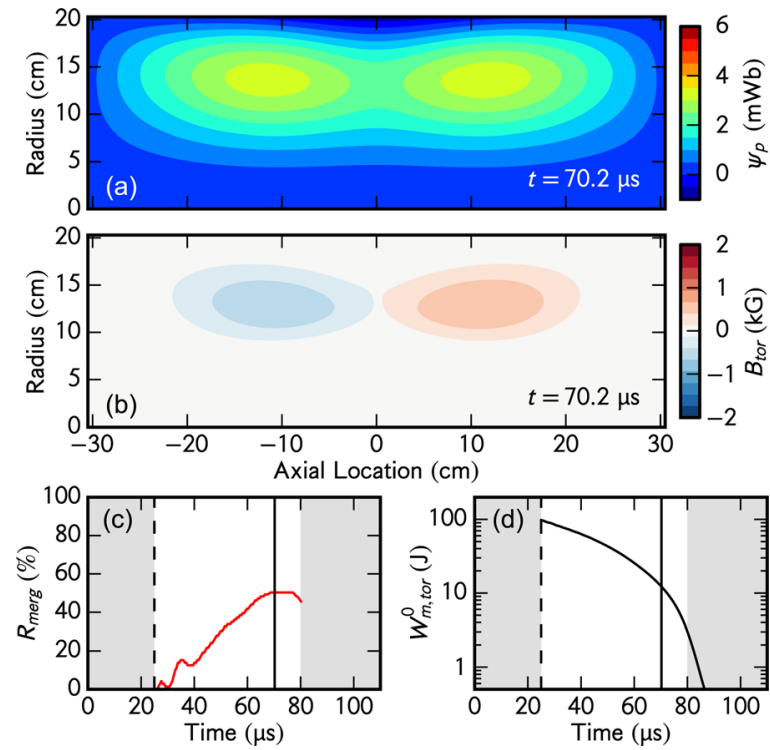

FIG. 5. (Color online) The plasma configuration that forms during the HYM-SSX simulations. (a) Contours of the $n=0$ poloidal flux $\psi_{p}$ at $t=70.2$ $\mu$ s after the merging process has stagnated. (b) Contours of the toroidal field at the same time showing that the these simulations produce a doublet CT. (c) Evolution of the merging fraction $R_{\text {merg }}$, which saturates at $R_{\text {merg }} \simeq 50 \%$. (d) Evolution of the total $n=0$ toroidal magnetic energy $W_{m, t o r}^{0}$ showing that significant toroidal field persists into the tilt phase of the simulation. 
The emergence of the tilt mode marks a transition to non-axisymmetric behavior in the simulations. As described in Sec. III A, non-axisymmetry is seeded by a turbulent initial velocity perturbation. Fig. 6 shows that this turbulent perturbation quickly organizes into coherent toroidal modes. The most virulent of these modes, the $n=1$ tilt mode, begins to grow almost immediately. The fitted linear growth time for this mode is $\gamma_{1}^{-1} \simeq 2.9 \mu \mathrm{s}\left(\gamma_{1} \simeq t_{A 0}^{-1}\right)$, which is consistent with the ideal MHD estimate ${ }^{26}$ of $\gamma_{1}=0.7-1.3 t_{A 0}^{-1}$. The tilt mode transitions to the nonlinear regime around $t=60 \mu \mathrm{s}$, which is around the time that it becomes experimentally detectable $\left(\sim 10^{-2} \mathrm{~kJ} / \mathrm{m}^{3}\right)$. Figure 6 also shows the development of the $n=2,3$ modes. The growth of these higher- $n$ modes is initially driven by numerical coupling to the $n=1$ tilt mode, though it later results from physical nonlinear coupling between the various modes.

The behavior of the SSX-like simulations is summarized in the pressure and magnetic field line plots of Fig. 7. These plots, which are produced with the Vislt software package, show (a) the axisymmetric initial conditions; (b) the partially merged doublet CT structure; and (c) the tilt-dominated structure late in time. In the initial condition plots $(t=25.0 \mu \mathrm{s})$, two field lines are used to trace out an irrational flux surface that is near the $q=1 / 3$ rational surface within in each spheromak. In the doublet CT plots $(t=62.4 \mu \mathrm{s})$, we see that the public regions of the doublet CT contain a non-negligible amount of confined plasma pressure. Despite this, the highest pressure contours (red) remain within the private flux regions at either end of the plasma. Note that the peak pressure $p_{\max }$ increases from the initial conditions due to Ohmic heating in the plasma. The corresponding $t=62.4 \mu$ s field line plot shows the magnetic topology of the doublet CT. The outer set of field lines is composed of 100 primarily poloidal field lines that trace out an FRC-like flux surface. In the figure, these shared field lines, which each close in a single turn, surround two additional flux surfaces that are still spheromak-like. It is this combination of FRC- and spheromak-like characteristics that defines the doublet CT configuration.

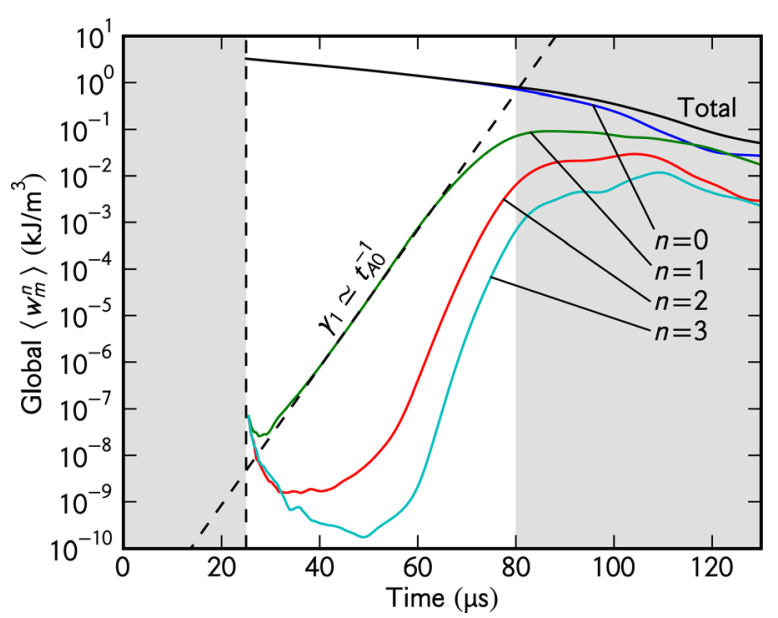

FIG. 6. (Color online) Time evolution of the various toroidal modes during the simulation. Each curve represents the globally averaged energy density $\left\langle w_{m}^{n}\right\rangle$ for a given toroidal mode number $n$. Note that the $n=1$ tilt mode is the most virulent mode in the simulation and that its fitted linear growth rate is $\gamma_{1}^{-1} \simeq 2.9 \mu \mathrm{s} \simeq t_{A 0}$.
Though the field lines within the doublet CT are initially closed, they eventually become stochastic during the nonaxisymmetric phase of the simulation. This behavior is manifest in the field line plot at $t=82.2 \mu \mathrm{s}$, where a single field line fills a stochastic volume that traverses throughout the configuration. Note that the two private flux regions have tilted in opposite directions, forming a butterfly like topology with $n=1$ character. Similar non-axisymmetric features are visible in the corresponding pressure plot.

\section{B. Comparison to experimental measurements}

The HYM-SSX simulation results described in Sec. IV A will now be compared directly to experimental data from SSX-FRC. To do this, magnetic measurements are extracted from the simulation data at locations that correspond to the magnetic probe locations in SSX-FRC. We then use the same analysis techniques that are applied to the experimental data in order to facilitate direct comparisons between the two. The first comparison concerns the mode energy density analysis introduced in Sec. II B. We begin by providing a more detailed description of this analysis procedure.

The first step in the magnetic energy density analysis is the toroidal mode decomposition of the vector magnetic field components $\left(B_{z}, B_{r}, B_{\theta}\right)$ that are acquired from each measurement location $\left(z_{i}, r_{j}, \theta_{k}\right)$. For $B_{z}$, the mode decomposition gives

$$
B_{z}^{n}\left(t \mid z_{i}, r_{j}\right)=\frac{1}{N_{\theta}}\left|\sum_{k=1}^{N_{\theta}} B_{z}\left(t \mid z_{i}, r_{j}, \theta_{k}\right) e^{-i n \theta_{k}}\right|
$$

where $i=1 \ldots 3, j=1 \ldots N_{r}$, and $k=1 \ldots N_{\theta}$. Note that in the experiments $N_{r}=8$ and $N_{\theta}=4$, while in the simulations $N_{r}=128$ and $N_{\theta}=32$. Also, we restrict the toroidal mode number to $n \geq 0$, so an extra factor of 2 must be included in Eq. (9) when $n>0$ to account for the contribution from the corresponding $n<0$ mode. After computing the Fourier amplitudes $\left(B_{z}^{n}, B_{r}^{n}, B_{\theta}^{n}\right)$, the localized poloidal and toroidal magnetic energy densities can be calculated at each $\left(z_{i}, r_{j}\right)$ location

$$
\begin{gathered}
w_{m, p o l}^{n}\left(t \mid z_{i}, r_{j}\right)=\frac{1}{2 \mu_{0}}\left[\left(B_{z}^{n}\right)^{2}+\left(B_{r}^{n}\right)^{2}\right], \\
w_{m, t o r}^{n}\left(t \mid z_{i}, r_{j}\right)=\frac{1}{2 \mu_{0}}\left(B_{\theta}^{n}\right)^{2} .
\end{gathered}
$$

The final step is to radially average these localized magnetic energy densities to reduce the data to one waveform per axial measurement location $z_{i}$ and toroidal mode number $n$

$$
\left\langle w_{m}^{n}\right\rangle\left(t \mid z_{i}\right)=\frac{2}{N_{r} R_{c}} \sum_{j=1}^{N_{r}} r_{j-1 / 2}\left[w_{m}^{n}\right]_{j-1 / 2},
$$

where

$$
\begin{gathered}
r_{j-1 / 2} \equiv \frac{1}{2}\left[r_{j}+r_{j-1}\right] \\
{\left[w_{m}^{n}\right]_{j-1 / 2} \equiv \frac{1}{2}\left[w_{m}^{n}\left(t \mid z_{i}, r_{j}\right)+w_{m}^{n}\left(t \mid z_{i}, r_{j-1}\right)\right] .}
\end{gathered}
$$



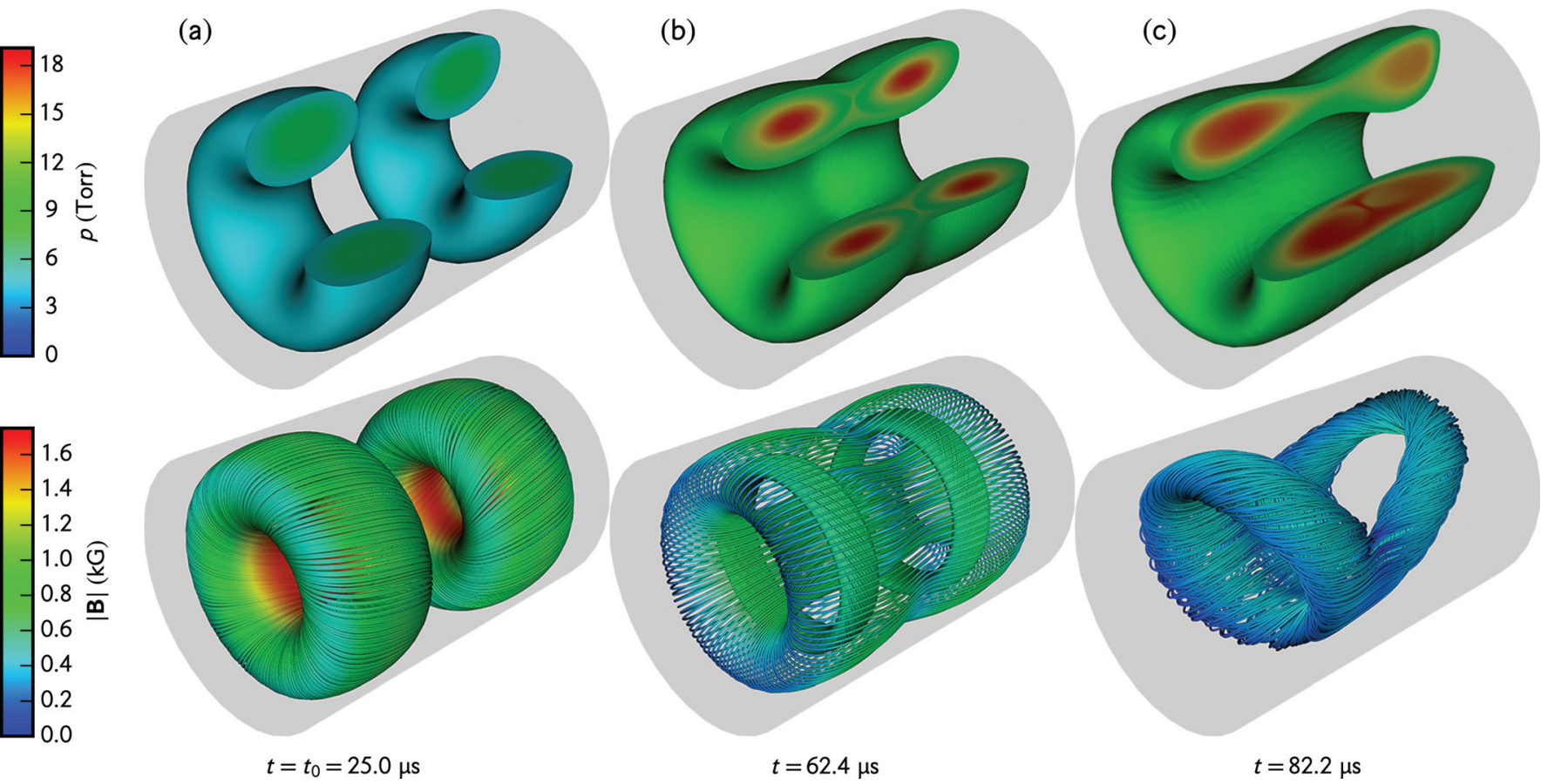

FIG. 7. (Color) Pressure and magnetic field line renderings of the simulated merging process. In each pressure plot, the displayed volume is bounded by the $p \simeq 0.5 p_{\max }$ surface. The $p_{\max }$ value changes with time due to Ohmic heating in the plasma. The field lines in the corresponding magnetic plots are seeded near the same $p \simeq 0.5 p_{\max }$ surface except where noted. (a) The initial conditions at $t=t_{0}=25.0 \mu \mathrm{s}$. In each spheromak, one long field line traces out an irrational flux surface. (b) The doublet CT configuration at $t=62.4 \mu \mathrm{s}$. The outer field lines are 100 individual field lines that trace out an FRC-like shared flux surface. The two inner spheromak-like flux surfaces are each traced out by a single long field line that is seeded near the $p \simeq 0.9 p_{\text {max }}$ surface. (c) The tilt-dominated, non-axisymmetric configuration at $t=82.2 \mu \mathrm{s}$. The magnetic field line plot now contains only a single field line that traverses throughout the butterfly shaped configuration.

Note that in the experiments, $\left[w_{m}^{n}\right]_{1 / 2}$ is approximated as $w_{m}^{n}\left(t \mid z_{i}, r_{1}\right)$ because there are no probe measurements on axis. In the simulations on the other hand, the $j=0$ mesh nodes are used to properly compute $\left[w_{m}^{n}\right]_{1 / 2}$. As in the SSX experimental analysis, the vacuum RCC magnetic energy density is subtracted from $w_{m, p o l}^{0}$.

The various $\left\langle w_{m, p o l}^{0}\right\rangle$ and $\left\langle w_{m, t o r}^{0}\right\rangle$ waveforms that are obtained from the above analysis of the simulation data are shown in bold in Fig. 8(a). The corresponding experimentally measured mode energies from Fig. 2 are reprinted in relief for comparison. It is clear that these two sets of waveforms have many features in common. This is especially true in subplot (i) of Fig. 8(a), which shows the evolution of the $n=0$ poloidal magnetic energy density. Both of the green midplane waveforms in this subplot indicate similar accumulation of poloidal magnetic energy as a result of reconnection between the two spheromaks. This behavior is followed by the stagnation of the merging process and the eventual rolloff of the $n=0$ waveforms late in time.

In subplot (ii) of Fig. 8(a), we find that there is qualitative but not quantitative agreement between the $n=0$ toroidal field waveforms. The measured toroidal field energy in the experiment is much stronger than the toroidal field energy found in the simulations at the same $z_{p r}= \pm 21.6 \mathrm{~cm}$ off-midplane probe locations. We hypothesize that this discrepancy is due to the fact that the formation and evolution of the plasma in the gun regions is not simulated. Thus, it is possible that the experimental plasma extends some distance back into the gun regions that are absent in the simulations. The simulated doublet CT configuration would then be axially shorter than the experimental doublet CT. We test this hypothesis by taking additional off-midplane probe measurements from the simulations, this time at $z_{p r}= \pm 15.6$ $\mathrm{cm}$ (Fig. 8(b)). This second set of measurements shows much better agreement between the $n=0$ toroidal field waveforms, which circumstantially supports the coaxial-guneffect hypothesis described here.

We can continue to investigate this hypothesis by looking, as we did in Fig. 3, at magnetic vector plots of the merged configuration. Figure 9 contains three sets of these magnetic vector plots, each showing the filtered $n=0$ magnetic structure from $t=62.4 \mu \mathrm{s}$. They are (a) from the experiments; (b) from the simulations with $z_{p r}= \pm 21.6 \mathrm{~cm}$; and (c) from the simulations with $z_{p r}= \pm 15.6 \mathrm{~cm}$. It is clear that the pitch and amplitude of the vectors in (b) do not closely resemble those from (a). In fact, the vectors in (b) are consistent with flux surfaces that are farther outboard (i.e., stronger $B_{r}$ and weaker $B_{\theta}$ ) than those in the experimental measurements. At the shifted off-midplane probe locations shown in (c), however, the vectors are in much better agreement with the experimental observations. This provides additional evidence that the simulated doublet CT is axially shorter than the experimental configuration.

It should be noted that the peak toroidal field amplitude in the simulations is smaller than the experimental measurements regardless of the measurement location $z_{p r}$. While both the temporal and spatial toroidal field profiles are qualitatively consistent (see Figs. 8 (b) (ii) and 9(a)/9(c), respectively), the amplitude discrepancy remains unexplained. In fact, additional toroidal field in the simulations upsets the 
(a) Mode energy density comparison $\left(z_{p r}= \pm 21.6 \mathrm{~cm}\right)$ :

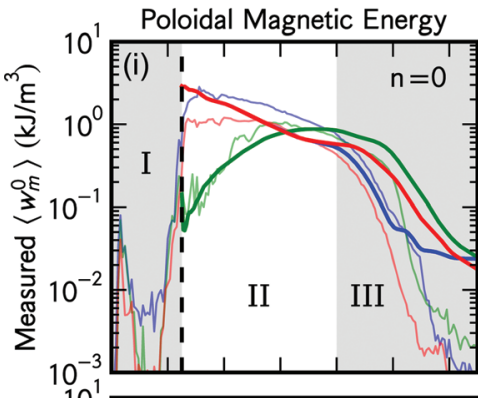

Toroidal Magnetic Energy
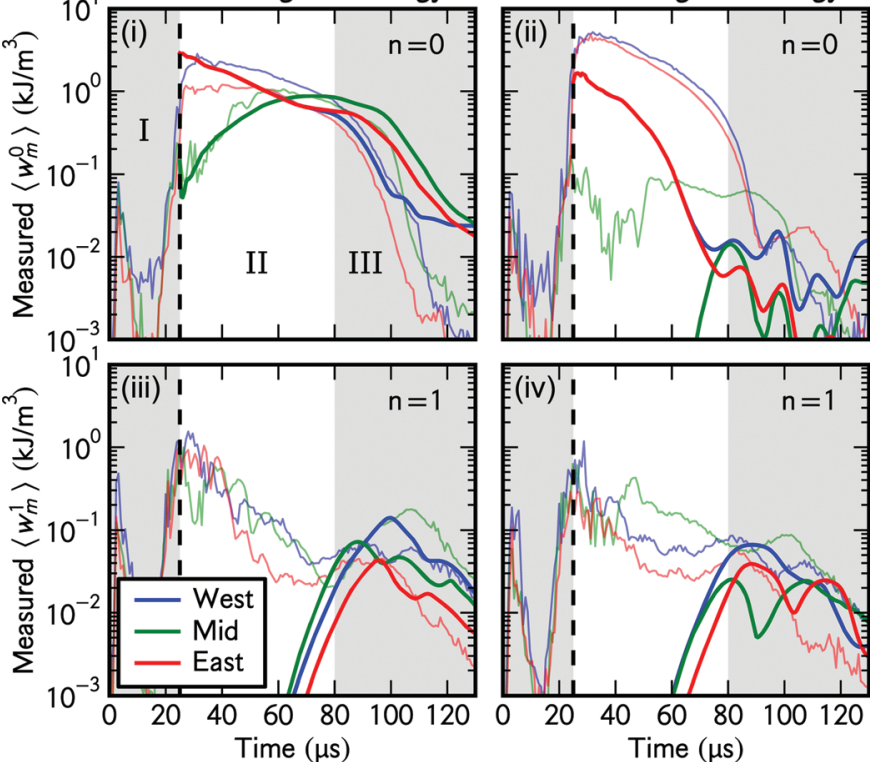

(b) Mode energy density comparison $\left(z_{p r}= \pm 15.6 \mathrm{~cm}\right)$ :

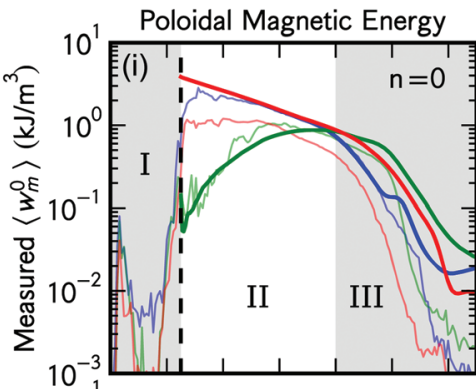

Toroidal Magnetic Energy
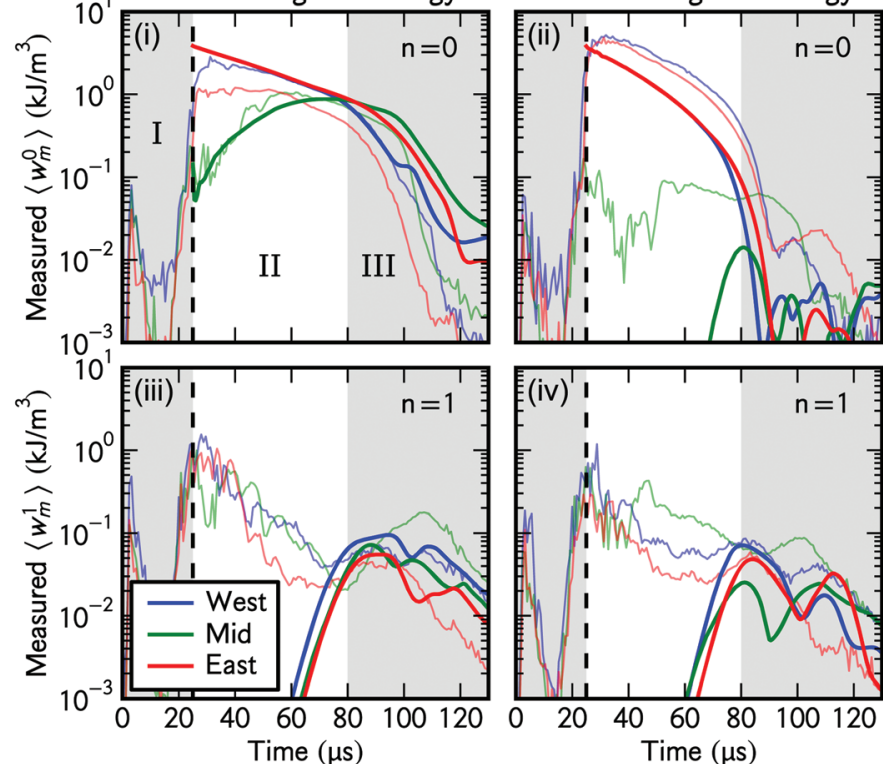

FIG. 8. (Color) Comparison between the radially averaged magnetic energy densities $\left\langle w_{m}^{n}\right\rangle$ extracted from the simulation data and those measured in SSX-FRC (see Fig. 2). Strong agreement is found with the accumulation of $n=0$ poloidal energy at the midplane due to reconnection and with the emergence of the $n=1$ tilt mode late in time. On the other hand, the $w_{m, t o r}^{0}$ waveforms in (a) agree only qualitatively. Much better agreement is found in (b) where the off-midplane measurements of the simulation data are taken at $z_{p r}= \pm 15.6 \mathrm{~cm}$ instead of $z_{p r}= \pm 21.6 \mathrm{~cm}$.

(a) Magnetic vectors $\left(\mathrm{n}=0\right.$; experiment; $\left.z_{\rho r}= \pm 21.6 \mathrm{~cm}\right)$ :

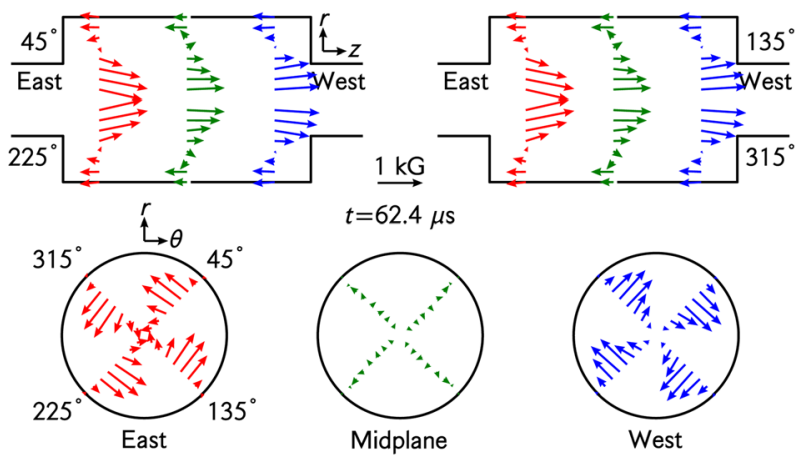

(b) Magnetic vectors $\left(\mathrm{n}=0\right.$; simulation; $\left.z_{p r}= \pm 21.6 \mathrm{~cm}\right)$ :

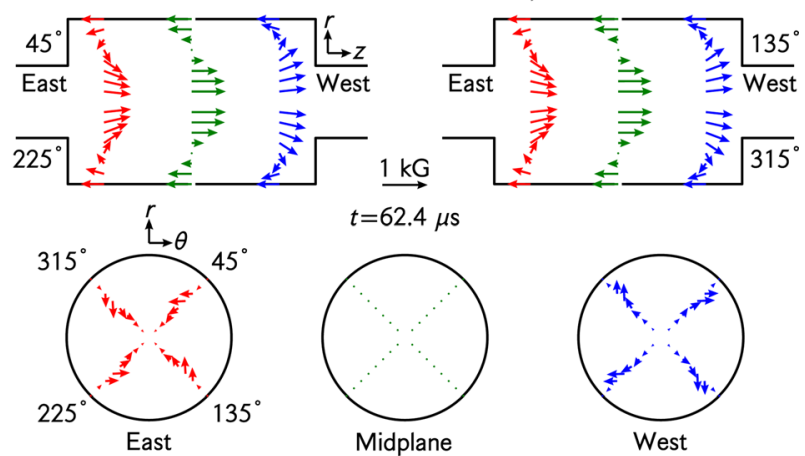

(c) Magnetic vectors $\left(\mathrm{n}=0\right.$; simulation; $\left.z_{p r}= \pm 15.6 \mathrm{~cm}\right)$ :

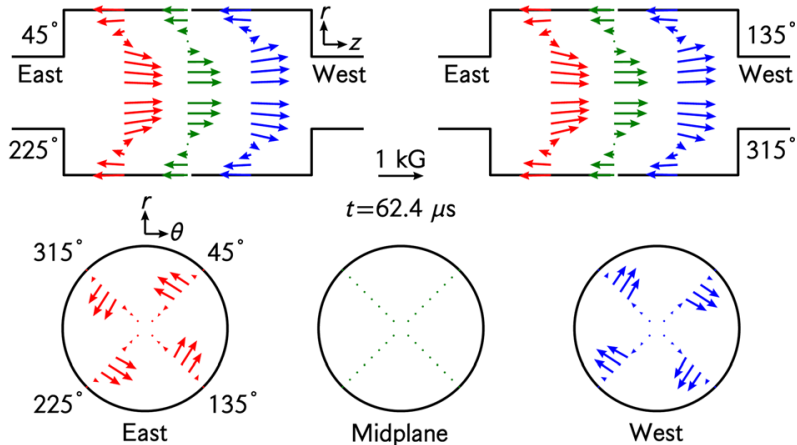

FIG. 9. (Color online) Comparison of magnetic vector plots from the experiment and the simulations. (a) Experimental $n=0$ vector plots reprinted from Fig. 3(b). (b) Analogous vector plots extracted from the simulations with the off-midplane probes at $z_{p r}= \pm 21.6 \mathrm{~cm}$, which is the same location as in the experiment. The pitch and amplitude of these vectors indicate that this probe location cuts across flux surfaces that are farther outboard than the ones measured in the experiments. (c) A different set of vector plots from the simulations with the off-midplane probes at $z_{p r}= \pm 15.6 \mathrm{~cm}$ instead. The structure of these plots is much more consistent with the experimental observations, indicating that effects from the coaxial magnetized guns, which are not included in the simulations, are likely important in this context. 
MHD quasi-equilibrium, so it is not clear how such large quantities of toroidal field are confined by the experimental plasma.

The final remaining element of the data comparisons in this section is to examine the behavior of the $n=1$ tilt mode. Returning to Fig. 8, it is clear that the nonlinear growth of the tilt mode in the simulations coincides with the experimentally measured increase in the $n=1$ energy density around $t \simeq 80 \mu \mathrm{s}$. In fact, the behavior of the $n=1$ mode is nearly identical at the two off-midplane probe locations. The experimentally measured $n=1$ energy prior to $t \simeq 80 \mu$ s is attributed to inherent non-axisymmetries that arise during the spheromak injection process. The conclusion, then, is that the tilt mode in the simulations, which grows linearly at small amplitude with $\gamma_{1}^{-1} \simeq t_{A 0}$, is entirely consistent with the slower experimentally observed mode that grows with $\gamma_{1}^{-1} \sim 6-8 t_{A 0}$ : it simply transitions into the slower-growing nonlinear regime before it is detected experimentally.

\section{Validity of the MHD model in the SSX regime}

The strong agreement between the HYM-SSX simulations and the SSX-FRC experiments that is demonstrated here clearly indicates that the resistive MHD model used for these simulations provides a good description of the global dynamics of the SSX-FRC plasmas. In order to verify that the resistive MHD model is valid in the SSX-FRC regime, an additional set of 2D simulations has been performed to study the effects of two-fluid physics on the observed merging dynamics. ${ }^{29}$ Overall, it is found that the global dynamics of the SSX-FRC merging process do not change substantially in the two fluid simulations. In particular, the reconnection timescales and the global magnetic field and plasma profiles are similar. Also, the magnitudes of the macroscopic flows are not strongly affected. This is consistent with the observation that the Hall MHD description of magnetic reconnection is appropriate when the condition $\lambda_{i} \gtrsim \delta_{S P}$ is satisfied, ${ }^{30}$ where $\delta_{S P}=L / \sqrt{S}$ is Sweet-Parker width of the reconnection current sheet and $\lambda_{i}$ is the ion skin depth. For the SSXFRC plasmas, $\lambda_{i} \lesssim \delta_{S P}$ such that the reconnection process is predominantly classical.

Some notable changes do occur in the two fluid simulations; these include a $\sim 25 \%$ increase in the peak reconnection rate and changes in the magnetic field and velocity profiles in the reconnection region. In particular, the radial component of the reconnection outflow velocity is enhanced in the two-fluid simulations. ${ }^{29}$ These changes are primarily due to the inclusion of Hall effects that produce the wellknown out-of-plane quadrupole field. This quadrupole field alters the local geometry of the reconnection layer from a quasi-2D MHD layer to a 3D layer with locally bent field lines. These findings are supported by experimental results that show that Hall effects modify the radial outflow profiles and radially shift the X-point during counter-helicity spheromak merging. ${ }^{31}$ It should be noted that the two-fluid simulations described here are computationally expensive and are therefore not used for the production-scale 3D simulations presented in this paper.

\section{RECONNECTION PHENOMENA}

Though the HYM-SSX simulations capture the global dynamics of spheromak merging in SSX-FRC, it is not clear that their applicability should extend to the reconnection region. Of concern are the aforementioned Hall and two-fluid effects whose impact on the reconnection dynamics is well known. Despite this, comparisons between the simulation data and experimental measurements again show respectable agreement (see Fig. 10). Here, field line reconstructions from a high density $5 \times 8 \times 5\left(N_{z} \times N_{r} \times N_{\theta}\right)$ magnetic probe array ${ }^{18,32}$ at the midplane of SSX are compared to field lines from the same region in the simulations. Note that the experiments with the 3D probe array were conducted in a larger flux conserver with $L / R=2.4(R=0.25 \mathrm{~m}$ and $L=0.60 \mathrm{~m})$ instead of $L / R=3$.

The field lines in Fig. 10 are compared at two times. The first is at $t=31.2 \mu$ s (Fig. 10(a)), which is shortly after the beginning of the merging process. In the two plots, the current sheet length $\left(\ell_{c s} \simeq 3 \mathrm{~cm}\right)$ and aspect ratio $\left(\ell_{c s} / \delta_{c s} \simeq 5\right)$ are very similar. At this early time, the reconnection is relatively two-dimensional $(z-r)$ because the reconnecting field lines from the edges of the two spheromaks are still mostly poloidal. The second set of field line plots (Fig. 10(b)) comes from the tilt phase of the discharge at $t=82.2 \mu \mathrm{s}$. Here, the remnants of the reconnection layer have tilted out of the local field line tracing region, leaving behind nearly poloidal field lines from the outer regions of the doublet CT. The simulation plot is again similar to the experiment, though it should be noted that the non-axisymmetry of the tilted configuration means that this plot shows one of several field line topologies that exist in the simulations at this time.

(a) Field line comparison $(t=31.2 \mu \mathrm{s})$ :
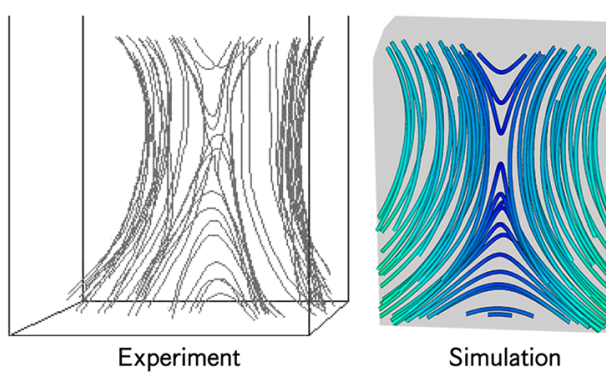

(b) Field line comparison $(t=82.2 \mu \mathrm{s})$ :
Experiment

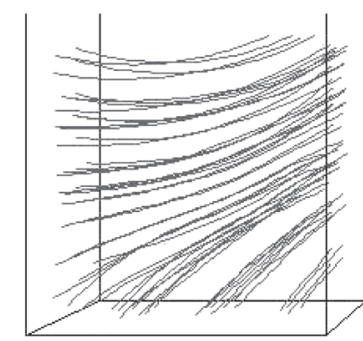

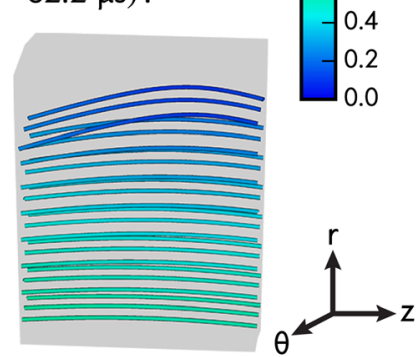

Simulation
FIG. 10. (Color online) Comparison of field lines in the reconnection region. The experimental field lines are reconstructed using measurements from a high density 3D magnetic probe array, which was implemented for merging experiments in a larger $L / R=2.4$ flux conserver. (a) Reconnecting field lines early in time. (b) Tilted field lines late in the discharge. 
We now proceed to analyze the dynamics of the simulated reconnection layer in more detail. Local current and velocity profiles from the simulations are shown in Fig. 11. Note that when the reconnection rate peaks around $t \simeq 55.2 \mu \mathrm{s}$, the reconnection current density is primarily poloidal (radial at the midplane) while the outflow velocity is instead primarily toroidal. If only poloidal field were reconnecting, the current sheet would be two dimensional with toroidal current density and radial outflows. As the counter-helicity spheromaks reconnect, however, increasing amounts of toroidal field enter the reconnection region, creating a current sheet that is obliquely inclined in the $r-\theta$ plane. Thus, the reconnection current density, which remains normal to the current sheet, will have both poloidal and toroidal components.

The oblique inclination of the current sheet also drives strongly sheared toroidal outflows on either side of the $\mathrm{X}$-line. In particular, the distribution of reconnecting poloidal and toroidal field creates field lines that enter the reconnection region with a sheared profile in the $r-\theta$ plane (see Fig. 12(d)). As the progression in Fig. 12 shows the tension in these sheared field lines is released as they reconnect and move away from the $\mathrm{X}$-line. Their ends snap straight thereby spinning up the plasma toroidally in opposite directions on opposite sides of the X-line. This so-called "slingshot effect" was first identified experimentally by Yamada et al. ${ }^{19}$ and is the primary driver of the toroidal flows found in these simulations. These toroidal flows do not, however, account for bursty radial outflows that have been observed in the SSXFRC experiments. ${ }^{33}$ In fact, the reconnection process is relatively quiescent throughout these simulations and does not produce any such impulsive events.

\section{SCAN OF VARIOUS PLASMA PARAMETERS}

In order to further investigate the physics of the counterhelicity merging process studied in this paper, we have carried out a simulation parameter scan over three key parameters: resistivity, viscosity, and line-tying. As shown in Fig. 13, moving one or more of these parameters out of the SSX-FRC regime can produce entirely different merging behaviors. The fully merged poloidal flux contours shown in Fig. 13(b) are from a simulation with lower resistivity $(S \simeq 1400)$ and no line-tying $\left(\zeta_{L T}=0.0 \%\right)$. When compared to the SSX-like simulation (Fig. 13(a)), we see that the merging fraction $R_{\text {merg }}$ approaches $100 \%$ instead of stagnating at $R_{\text {merg }} \simeq 50 \%$ (Fig. 13(c)). Also, the completed merging process annihilates all of the toroidal field energy $W_{m \text {.tor }}^{0}$ before the tilt mode emerges in the simulation (Fig. 13(d)), leaving behind an axisymmetric, toroidal-field-free FRC.

The data shown in Fig. 13 comes from just 2 of the 61 data points in the aforementioned multi-dimensional parameter scan. The scan covers three $2 \mathrm{D}$ cuts of the $S \times \operatorname{Re} \times \zeta_{L T}$ parameter space with each cut centered about the SSX-like simulation $\left(S=700, \mathrm{Re}=700\right.$, and $\left.\zeta_{L T}=4.7 \%\right)$. Figure 14 summarizes the results of the scan with contour plots of the merging fraction $R_{\text {merg }}$ and an additional simulation metric $\tau_{\text {stab }}$. The "stable lifetime" $\tau_{\text {stab }}$ systematically characterizes the relative stability of a given simulated plasma regardless of the nonlinear details of its tilt mode evolution. We define the stable lifetime $\tau_{\text {stab }}$ as

$$
\tau_{s t a b} \equiv t_{10 \%}^{1}-t_{0},
$$

where $t_{10 \%}^{1}$ is the time when the amount of magnetic energy in the $n=1$ mode becomes more than $10 \%$ of the energy in the $n=0$ mode $\left(W_{m}^{1} / W_{m}^{0} \geq 10 \%\right)$. Thus, $\tau_{s t a b}$ represents the length of time over which the configuration is predominantly axisymmetric.

In the parameter scan data shown in Fig. 14, we find several interesting trends. Simulations with complete merging $\left(R_{\text {merg }} \rightarrow 100 \%\right)$ are found only in the extrema of the scan, particularly in the low-resistivity, low-line-tying limit.
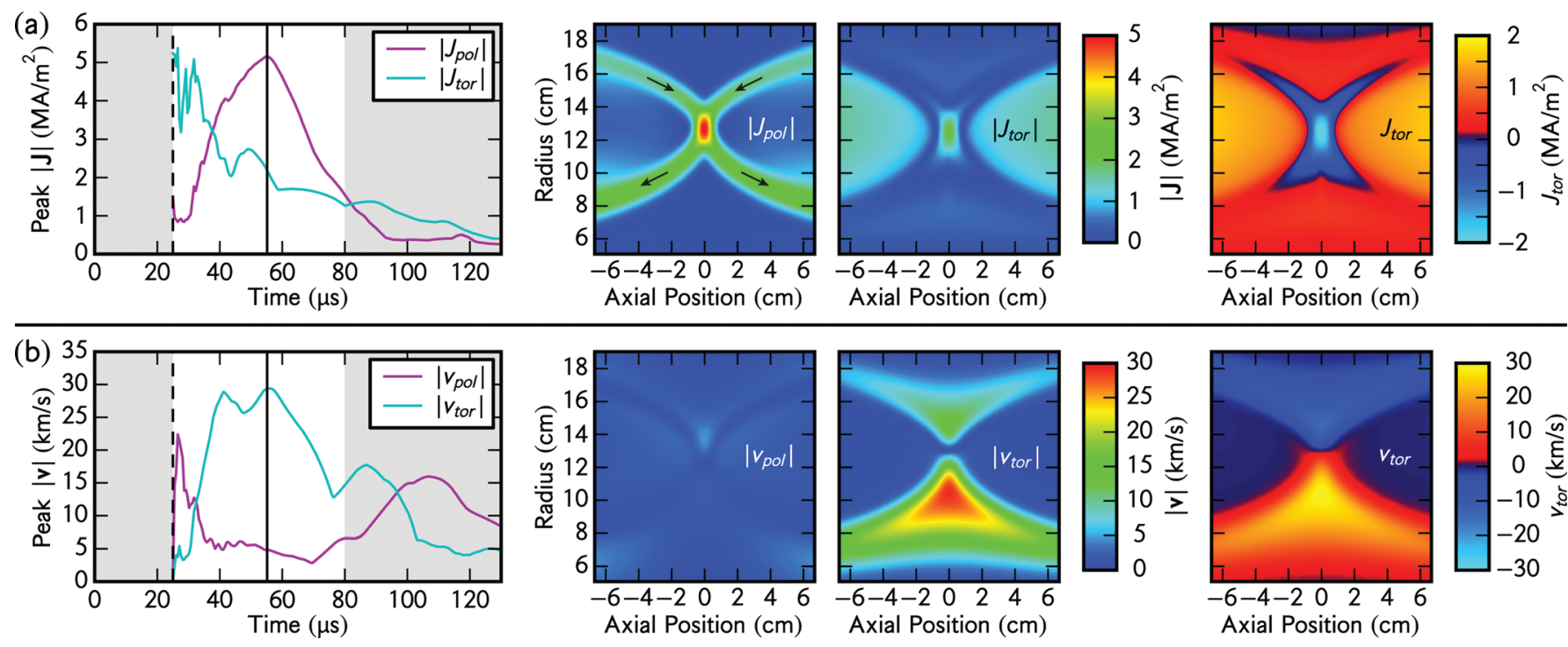

FIG. 11. (Color) Reconnection region profiles of (a) current density and (b) flow velocity. Each plot on the far left contains the time evolution of the peak poloidal and toroidal components of the relevant quantity (i.e., $\mathbf{J}$ or $\mathbf{v}$ ). Note that the peak current density is primarily poloidal, while the peak flow is instead primarily toroidal. The psuedocolor plots in the middle compare the magnitudes of the poloidal and toroidal components of these quantities at $t=55.2 \mu \mathrm{s}$. Additional plots on the far right examine the shear in the toroidal component of each quantity. 

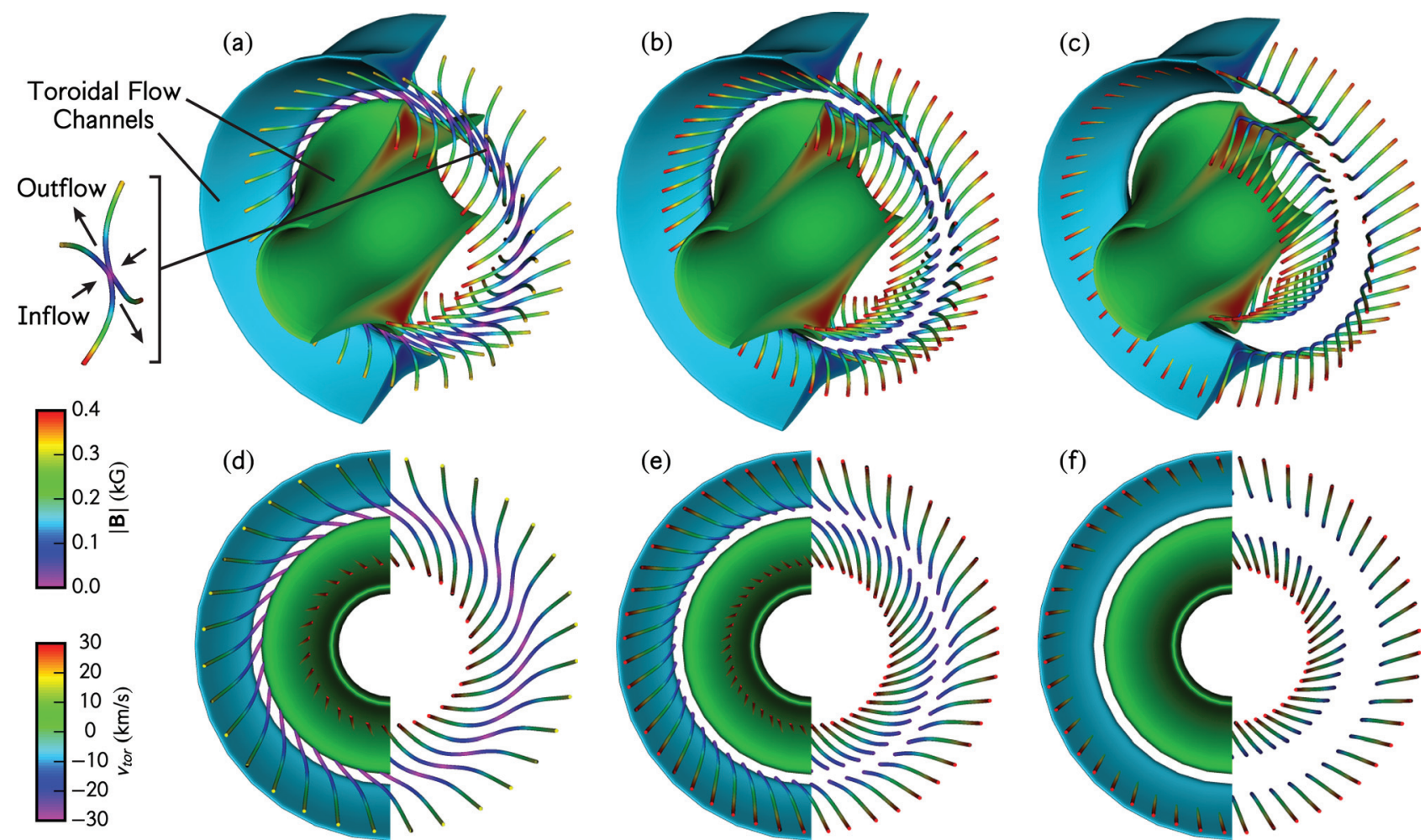

FIG. 12. (Color) Three-dimensional plots of the reconnection region at $t=55.2 \mu \mathrm{s}$. The plots in the bottom row are the $r-\theta$ projections of the corresponding isometric plots in the top row. Moving from left to right, each plot shows a series of reconnecting field lines that are progressively further away from the $\mathrm{X}$-line in the outflow region. The release of the tension in the sheared field lines following reconnection is visible as they snap straight during this progression. The snapping motion "slingshots" the plasma toroidally in opposite directions on opposite sides of the X-line.
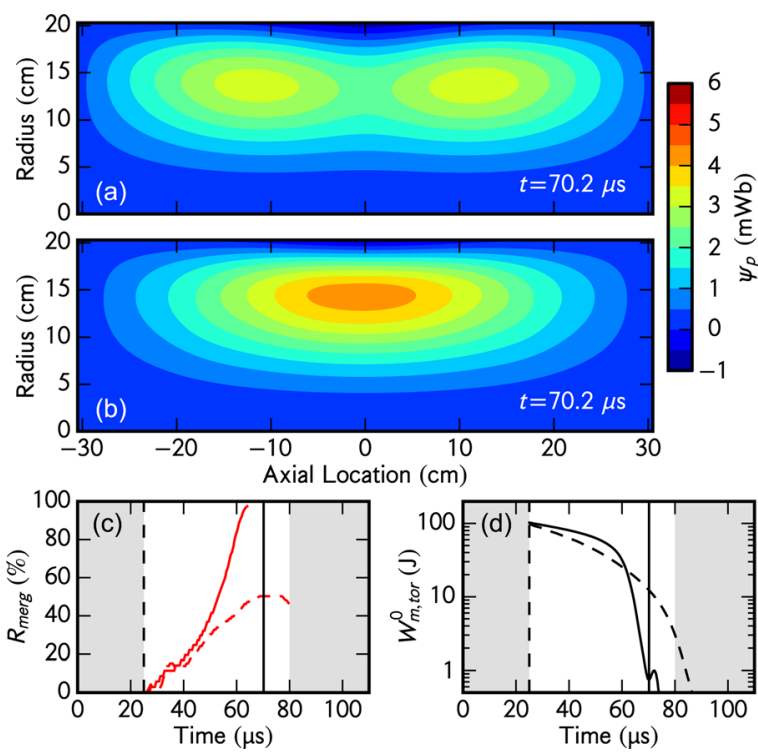

FIG. 13. (Color online) Comparison between a fully merging simulation and the SSX-like simulation. The SSX-like simulation in (a) has $S=700 \%$, $\operatorname{Re}=700 \%$, and $\zeta_{L T}=4.7 \%$, while the fully merging simulation in (b) has $S=1400 \%, \operatorname{Re}=700 \%$, and $\zeta_{L T}=0.0 \%$. With these parameter changes, the doublet CT structure is replaced by a fully merged FRC. (c) A comparison of the merging fractions $R_{\text {merg }}$ shows that $R_{\text {merg }} \rightarrow 100 \%$ for the fully merging simulation (solid line), while it stagnates at $R_{\text {merg }} \simeq 50 \%$ for the SSXlike simulation (dashed line). (d) Concurrently, the toroidal field energy $w_{m, t o r}^{0}$ rolls off much earlier for the fully merging simulation (solid line) than for the SSX-like simulation (dashed line).
High resistivity (small $S$ ), high viscosity (small Re), and high line-tying (large $\zeta_{L T}$ ) are each found to inhibit the merging process. With regard to stability, resistivity is found to have the most significant effect. At high resistivity, the configuration decays so quickly that the growth of the tilt instability becomes nonlinear at small amplitude. This leads to simulations with very long $\tau_{\text {stab }}$ for $S \lesssim 500$. High viscosity and high line-tying are also found to promote stability, though to a lesser extent than high resistivity. These results indicate that a wide variety of merging behaviors are accessible in the $S \times \operatorname{Re} \times \zeta_{L T}$ parameter space. Thus, we conclude that the specific location of a given experimental device in the broader parameter space of counter-helicity merging is very important. This implies that experimental details such as the plasma formation technique and the geometry of the flux conserver can have far reaching impacts on the behavior of the merging process.

\section{SUMMARY}

In this paper, we present $3 \mathrm{D}$ resistive MHD simulations that explore unexpected results from counter-helicity spheromak merging experiments in the SSX-FRC device. The simulations, which are highly consistent with experimental measurements, show that a partially merged "doublet CT" configuration forms instead of the anticipated toroidal-fieldfree FRC. This doublet CT has both FRC- and spheromaklike characteristics that produce an antisymmetric toroidal field profile. We also find that the experimentally observed slowly emerging $n=1$ tilt mode is consistent with the 

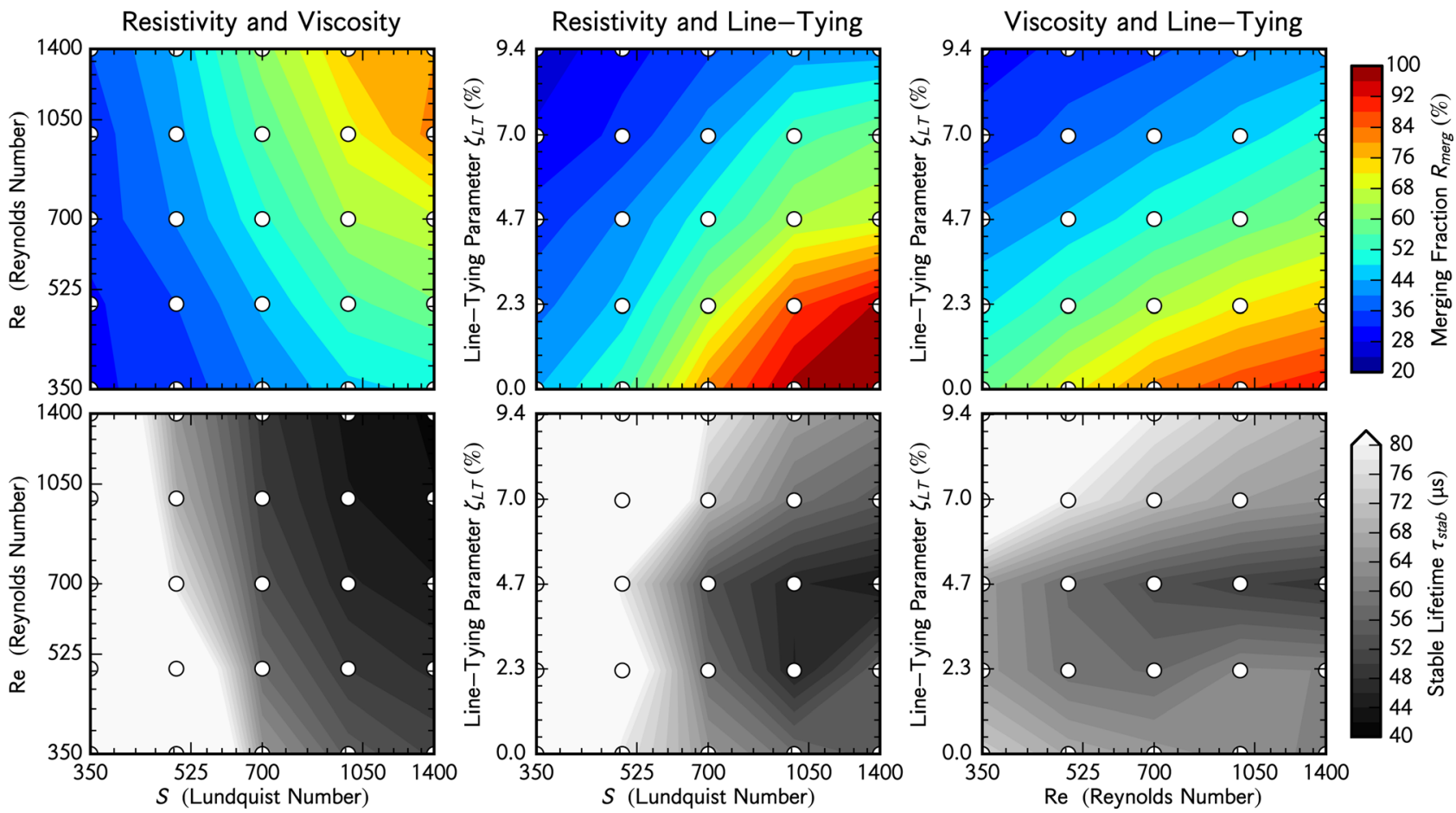

FIG. 14. (Color online) Contour plots of the merging fraction $R_{\text {merg }}$ and the stable lifetime $\tau_{\text {stab }}$ extracted from the 61-point simulation parameter scan. Each of the three sets of plots is a 2D cut of the $S \times \operatorname{Re} \times \zeta_{L T}$ parameter space and the center of each cut corresponds to the SSX-like simulation with $S=700$, $\operatorname{Re}=700$, and $\zeta_{L T}=4.7 \%$. Complete merging is found at low resisitivty and line-tying. High resistivity (small $S$ ) inhibits merging and promotes stability. High viscosity (small Re) and high line-tying (large $\zeta_{L T}$ ) also inhibit merging, but they have a more limited effect on the plasma stability than high resistivity.

nonlinear phase of the tilt mode in the simulations. The mode is found to grow linearly at small amplitude with $\gamma_{1} \simeq t_{A 0}^{-1}$ before becoming experimentally detectable. Investigation of reconnection phenomena in the simulations shows that large sheared toroidal outflows are driven by the "slingshot effect" that occurs due to the counter-helicity reconnection topology. Finally, a simulation parameter scan over resistivity, viscosity, and line-tying shows that a wide range of merging behaviors are possible, including full merging at lower resistivity and line-tying. These results indicate that the parameter space for counter-helicity spheromak merging is quite large and that diverse merging behaviors are to be expected in different devices.

\section{ACKNOWLEDGMENTS}

The authors gratefully acknowledge the technical contributions of D. Cohen, V. Chaplin, S. Palmer, and J. Haldeman and thank V. S. Lukin and J. B. Parker for useful discussions. This work is supported by Contract No. DE-AC0209CH11466 with the U.S. Department of Energy and by the Center for Magnetic Self-Organization (CMSO), a National Science Foundation (NSF) Frontier Center established in coordination with the Department of Energy.

\section{APPENDIX: ADDITIONAL SSX-FRC EXPERIMENTAL RESULTS}

In order to better constrain the HYM simulations presented in this paper, we have performed line-averaged experimental measurements of plasma density, ion temperature, and emissivity. Our conclusions, shown earlier in Table I, are that the SSX-FRC plasmas are in the MHD regime with peak density $n_{e}=10^{15} \mathrm{~cm}^{-3}$ and Alfvén speed of $70 \mathrm{~km} / \mathrm{s}$. The minimum ion skin depth is $c / \omega_{p i}=0.7 \mathrm{~cm}$. Temperatures are in the range of $20-50 \mathrm{eV}$, indicating relatively low resistivity.

Line-averaged ion flow and temperature $T_{i}$ at the midplane is monitored with a $1.33 \mathrm{~m}$ ion Doppler spectrometer (IDS). We achieve high spectral resolution $(0.0075 \mathrm{~nm}$ per pixel) with an Echelle grating operating at 25 th order. ${ }^{34} \mathrm{We}$ achieve high temporal resolution using a 32 channel photomultipler tube array. The SSX IDS instrument measures

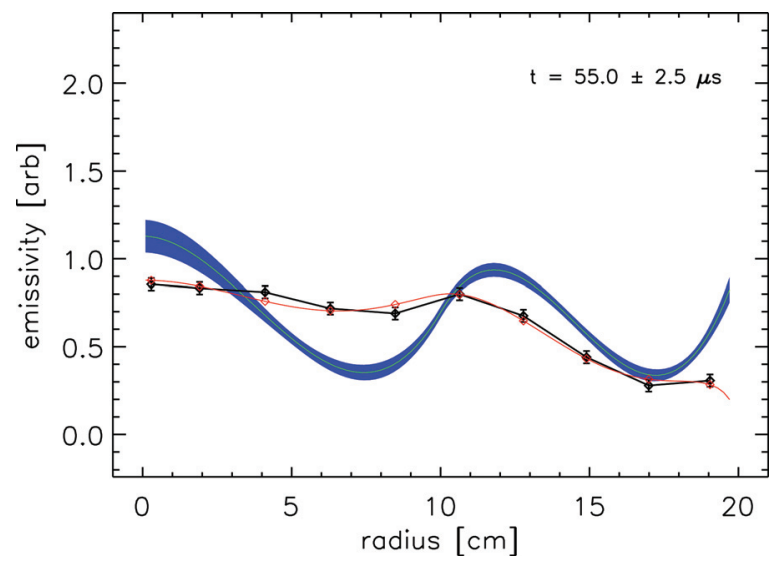

FIG. 15. (Color) Radial profile of total emissivity at $t \simeq 55 \mu$ s. A spline fit of measured emissivity (black curve with error bars) is Abel inverted to generate a plot of the emissivity as a function of radius (blue curve with blue band for error bars). The emissivity profile as a function of impact parameter is reconstructed from the Abel inversion (red smooth curve). Note the enhanced emissivity at $r=0$ and $r=12 \mathrm{~cm}$. 


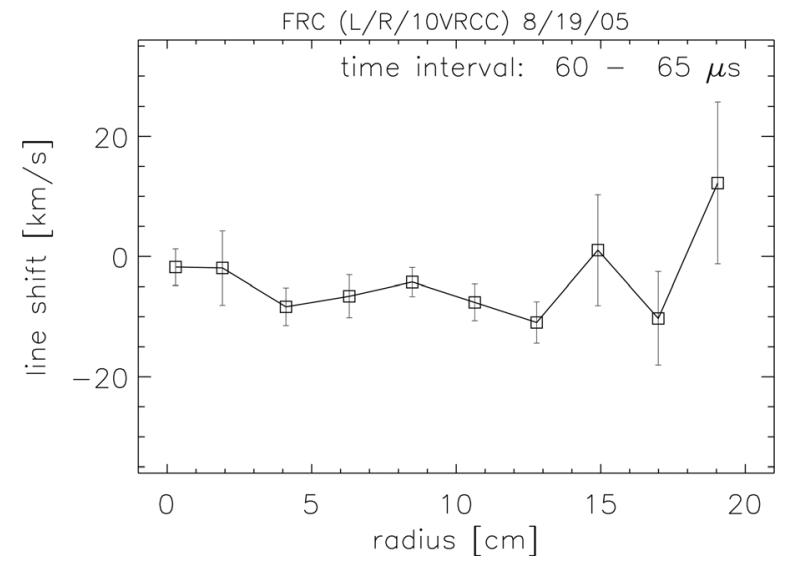

FIG. 16. IDS flow measurement as a function of impact parameter. Plotted is the line of sight velocity for chords with impact parameters between $r=0$ and $r=20 \mathrm{~cm}$. The purely radial flow near $r=0 \mathrm{~cm}$ is consistent with $v_{r}=0 \mathrm{~km} / \mathrm{s}$.

with $\leq 1 \mu$ s time resolution the width and Doppler shift of the $C_{I I I}$ impurity $229.687 \mathrm{~nm}$ line to determine the temperature and line-averaged flow velocity during spheromak merging events. Velocity resolution (obtained by fitting the lineshape) depends on both signal strength and the thermal Doppler width, but it can be as good as a few $\mathrm{km} / \mathrm{s} \mathrm{(a} \mathrm{few}$ percent of the Alfvén speed); the full width half maximum (FWHM) of the instrument function corresponds to $5 \mathrm{~km} / \mathrm{s}$ (3.4 eV for $C$ ions). In addition to the IDS measurements, energetic ions have been directly measured with electrostatic probes in SSX. ${ }^{35,36}$

Using the IDS, we can measure line shapes along a number of chords across the device at the midplane. In Fig. 15, we plot the total emissivity of the $C_{I I I}$ line as a function of impact parameter for 10 different chords. The intensity of the light is integrated across the entire line and 10 shots are averaged at each chord. Data is also averaged into $5 \mu$ s time bins to smooth shot-to-shot variability (black curve with data and error bars). A cubic spline is then fit to the data. Two matched splines are used, one that vanishes at the wall and another with vanishing derivative at $r=0$. The spline fit is Abel-inverted to generate a plot of the emissivity as a function of radius (blue curve with blue band for error bars).
Finally, the Abel inverted data can be used to re-construct the emissivity profile as a function of impact parameter (red smooth curve). If the inversion is correct, then the reconstruction should overlie the original data. For the data presented in Fig. 15, the fit is excellent.

Note that at the time depicted $(t=55 \mu \mathrm{s})$, the $C_{I I I}$ emission is enhanced at $r=0$ and $r=12 \mathrm{~cm}$. Magnetic data show that at the midplane, the magnetic axis is at $r=14 \mathrm{~cm}^{18}$ where we expect the particle confinement to be best and the plasma the hottest. For comparison, earlier in time during the reconnection process, emission is a factor of two higher and comes primarily from the core of the plasma (around $r=12$ $\mathrm{cm})$. From magnetic measurements, we know that at $t=55$ $\mu$ s, reconnection dynamics have settled down but the plasma is still axisymmetric. Abel inversion requires that the data be close to axisymmetric. This measurement indicates that emissivity (related to $n_{e}^{2}$ and $T_{e}$ ) varies only by a factor of two across the plasma radius late in time and that $C_{I I I}$ is a good tracer of bulk properties (not just edge effects). We find that emissivity is highest immediately after the spheromak merging process (around 40-50 $\mu \mathrm{s}$ ) and low during early formation and late decay.

In Fig. 16, we show a plot of the line-of-sight velocity profile as a function of impact parameter. Data from 10 shots are averaged at each chord in $5 \mu$ s bins. For the chord labeled $r=0 \mathrm{~cm}$, the component of the line-of-sight velocity is $v_{r}$ and our measurement is consistent with $v_{r}=0 \mathrm{~km} / \mathrm{s}$ there (as it should be). Note that at the time depicted $(t=60 \mu \mathrm{s})$, the data show that in general, the line-of-sight velocity is small $(\leq 5 \mathrm{~km} / \mathrm{s}$ ) across the radius of the plasma and that there is little or no shear. There is some indication of toroidal flow at $r=19 \mathrm{~cm}$ (line-of-sight velocity is mostly $v_{\theta}$ there). Early in time, we have observed high velocity outflows $( \pm 40 \mathrm{~km} / \mathrm{s})$ associated with reconnection. ${ }^{33}$ Note also that an Abel inversion of this velocity data would be difficult since the line of sight velocity consists of a different combination of $v_{r}$ and $v_{\theta}$ at each chord and $v_{z}$ is not measured.

Line-averaged density is measured with a quadrature $\mathrm{HeNe}$ interferometer. A modified Mach-Zehnder interferometer uses a linearly polarized scene beam and a circularly polarized reference beam to generate signals proportional to the sine and cosine of the phase shift introduced by the
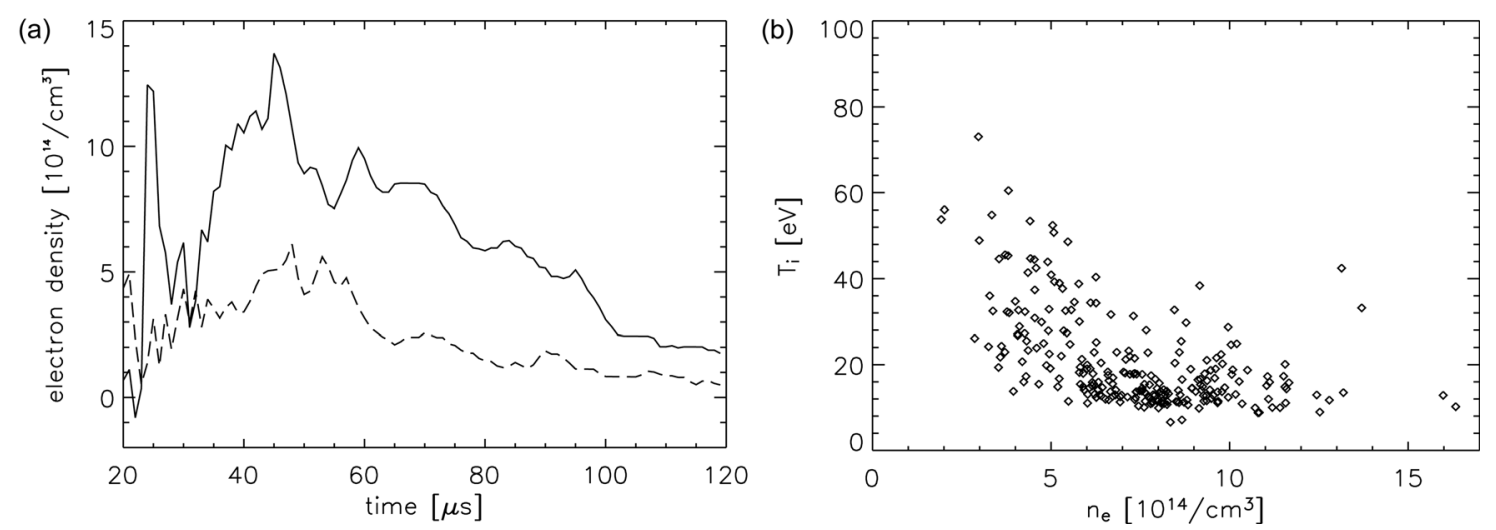

FIG. 17. Density measurements for counter-helicity merging in SSX-FRC. (a) A pair of density traces for two gas valve timings shows the range of accessible densities. (b) A scatter plot of $T_{i}$ vs. $n_{e}$ for various discharges. During the axisymmetric phase of the discharge, the typical density is $2-5 \times 10^{14} \mathrm{~cm}^{-3}$ and $T_{i}=20-40 \mathrm{eV}$ 
plasma. ${ }^{37}$ We are able to vary plasma density by adjusting gas valve timing while limiting recycling off the walls with baking and glow discharge conditioning. In Fig. 17(a), we plot a pair of density traces for different gas valve timings. Plasma densities range from $1-10 \times 10^{14} \mathrm{~cm}^{-3}$. In Fig. 17(b), we show a scatter plot of ion temperatures (from gaussian fits of IDS data) measured at a variety of plasma densities. Temperature and density are measured simultaneously (within a $10 \mu$ s window) immediately after the doublet CT is formed. Because optical access is limited at the midplane, densities were measured on the west end of the machine while ion Doppler measurements were performed at the midplane. Note that while there is a large scatter in the data, we obtain our highest ion temperatures at the lowest plasma densities. During the axisymmetric doublet CT phase discussed in this paper, the typical density is $2-5 \times 10^{14}$ $\mathrm{cm}^{-3}$ and $T_{i}=20-40 \mathrm{eV}$. Line averaged electron temperature is inferred from a model fit to data from a soft x-ray array as well as a vacuum ultraviolet spectrometer. We found $T_{e}$ increases from $10 \mathrm{eV}$ to $20 \mathrm{eV}$ after reconnection. ${ }^{38}$

${ }^{1}$ M. R. Brown, Phys. Plasmas 6, 1717 (1999).

${ }^{2}$ T. R. Jarboe, Plasma Phys. Controlled Fusion 36, 945 (1994).

${ }^{3}$ M. Tuszewski, Nucl. Fusion 28, 2033 (1988).

${ }^{4}$ M. Tuszewski, W. T. Armstrong, R. E. Chrien, W. N. Hugrass, K. F. McKenna, D. J. Rej, R. E. Siemon, D. P. Taggart, and B. L. Wright, Phys. Fluids B 3, 2844 (1991).

${ }^{5}$ M. Tuszewski, D. P. Taggart, R. E. Chrien, D. J. Rej, R. E. Siemon, and B. L. Wright, Phys. Fluids B 3, 2856 (1991).

${ }^{6}$ L. C. Steinhauer, Phys. Plasmas 18, 070501 (2011).

${ }^{7}$ S. A. Cohen, B. Berlinger, C. Brunkhorst, A. Brooks, N. Ferraro, D. Lundberg, A. Roach, and A. H. Glasser, Phys. Rev. Lett. 98, 072508 (2007).

${ }^{8}$ K. E. Miller, J. A. Grossnickle, R. D. Brooks, C. L. Deards, T. E. DeHart, M. Dellinger, M. B. Fishburn, H. Y. Guo, B. Hansen, J. W. Hayward, A. L. Hoffman, W. S. Kimball, K. Y. Lee, D. E. Lotz, P. A. Melnik, R. D. Milroy, Z. A. Pietrzyk, G. C. Vlasses, F. S. Ohuchi, and A. Tankut, Fusion Sci. Technol. 54, 946 (2008).

${ }^{9}$ T. R. Jarboe, I. Henins, H. W. Hoida, R. K. Linford, J. Marshall, D. A. Platts, and A. R. Sherwood, Phys. Rev. Lett. 45, 1264 (1980).

${ }^{10}$ E. V. Belova, R. C. Davidson, H. Ji, and M. Yamada, Phys. Plasmas 11, 2523 (2004).

${ }^{11}$ E. V. Belova, R. C. Davidson, H. Ji, and M. Yamada, Phys. Plasmas 13, 056115 (2006).

${ }^{12}$ M. Tuszewski, D. C. Barnes, R. E. Chrien, J. W. Cobb, D. J. Rej, R. E. Siemon, D. P. Taggart, and B. L. Wright, Phys. Rev. Lett. 66, 711 (1991).

${ }^{13}$ C. G. R. Geddes, T. W. Kornack, and M. R. Brown, Phys. Plasmas 5, 1027 (1998).

${ }^{14}$ H. S. McLean, R. D. Wood, B. I. Cohen, E. B. Hooper, D. N. Hill, J. M. Moller, C. Romero-Talamas, and S. Woodruff, Phys. Plasmas 13, 056105 (2006).
${ }^{15}$ P. M. Bellan, Spheromaks (Imperial College Press, London, 2000).

${ }^{16}$ A. Bondeson, G. Marklin, Z. G. An, H. H. Chen, Y. C. Lee, and C. S. Liu, Phys. Fluids 24, 1682 (1981).

${ }^{17}$ J. M. Finn, W. M. Manheimer, and E. Ott, Phys. Fluids 24, 1336 (1981).

${ }^{18}$ C. D. Cothran, A. Falk, A. Fefferman, M. Landreman, M. R. Brown, and M. J. Schaffer, Phys. Plasmas 10, 1748 (2003).

${ }^{19}$ M. Yamada, Y. Ono, A. Hayakawa, M. Katsurai, and F. W. Perkins, Phys. Rev. Lett. 65, 721 (1990).

${ }^{20}$ Y. Ono, M. Yamada, T. Akao, T. Tajima, and R. Matsumoto, Phys. Rev. Lett. 76, 3328 (1996).

${ }^{21}$ E. Kawamori and Y. Ono, Phys. Rev. Lett. 95, 085003 (2005).

${ }^{22}$ S. P. Gerhardt, E. Belova, M. Yamada, H. Ji, M. Inomoto, Y. Ren, and B. McGeehan, Phys. Rev. Lett. 99, 245003 (2007).

${ }^{23}$ M. W. Binderbauer, H. Y. Guo, M. Tuszewski, S. Putvinski, L. Sevier, D. Barnes, N. Rostoker, M. G. Anderson, R. Andow, L. Bonelli, F. Brandi, R. Brown, D. Q. Bui, V. Bystritskii, F. Ceccherini, R. Clary, A. H. Cheung, K. D. Conroy, B. H. Deng, S. A. Dettrick, J. D. Douglass, P. Feng, L. Galeotti, E. Garate, F. Giammanco, F. J. Glass, O. Gornostaeva, H. Gota, D. Gupta, S. Gupta, J. S. Kinley, K. Knapp, S. Korepanov, M. Hollins, I. Isakov, V. A. Jose, X. L. Li, Y. Luo, P. Marsili, R. Mendoza, M. Meekins, Y. Mok, A. Necas, E. Paganini, F. Pegoraro, R. Pousa-Hijos, S. Primavera, E. Ruskov, A. Qerushi, L. Schmitz, J. H. Schroeder, A. Sibley, A. Smirnov, Y. Song, X. Sun, M. C. Thompson, A. D. Van Drie, J. K. Walters, M. D. Wyman, and the TAE Team, Phys. Rev. Lett. 105, 045003 (2010).

${ }^{24}$ Y. A. Omelchenko, Phys. Plasmas 7, 1443 (2000).

${ }^{25}$ Y. A. Omelchenko, M. J. Schaffer, and P. B. Parks, Phys. Plasmas 8, 4463 (2001).

${ }^{26}$ E. V. Belova, R. C. Davidson, H. Ji, M. Yamada, C. D. Cothran, M. R. Brown, and M. J. Schaffer, Nucl. Fusion 46, 162 (2006).

${ }^{27}$ E. Belova, S. C. Jardin, H. Ji, M. Yamada, and R. Kulsrud, Phys. Plasmas 7, 4996 (2000).

${ }^{28}$ M. R. Brown, C. D. Cothran, J. Fung, M. Chang, J. Horwitz, M. J. Schaffer, J. Leuer, and E. V. Belova, Phys. Plasmas 13, 102503 (2006).

${ }^{29}$ E. V. Belova, R. C. Davidson, H. Ji, M. Yamada, S. P. Gerhardt, and M. Inomoto, Bull. Am. Phys. Soc. 50, 246 (2005).

${ }^{30}$ M. Yamada, Phys. Plasmas 14, 058102 (2007).

${ }^{31}$ M. Inomoto, S. P. Gerhardt, M. Yamada, H. Ji, E. Belova, A. Kuritsyn, and Y. Ren, Phys. Rev. Lett. 97, 135002 (2006).

${ }^{32}$ M. Landreman, C. D. Cothran, M. R. Brown, M. Kostora, and J. T. Slough, Rev. Sci. Instrum. 74, 2361 (2003).

${ }^{33}$ M. R. Brown, C. D. Cothran, C. E. Myers, E. V. Belova, and T. Gray, "Spectroscopic observation of bi-directional reconnection outflows in a laboratory plasma," Phys. Rev. Lett. (unpublished).

${ }^{34}$ C. D. Cothran, J. Fung, M. R. Brown, and M. J. Schaffer, Rev. Sci. Instrum. 77, 063504 (2006).

${ }^{35}$ M. R. Brown, C. D. Cothran, M. Landreman, D. Schlossberg, and W. H. Matthaeus, Astrophys. J. 577, L63 (2002).

${ }^{36}$ M. R. Brown, C. D. Cothran, M. Landreman, D. Schlossberg, W. H. Matthaeus, G. Qin, V. S. Lukin, and T. Gray, Phys. Plasmas 9, 2077 (2002).

${ }^{37}$ C. J. Buchenauer and A. R. Jacobsen, Rev. Sci. Instrum. 48, 769 (1977).

${ }^{38}$ V. H. Chaplin, M. R. Brown, D. H. Cohen, T. Gray, and C. D. Cothran, Phys. Plasmas 16, 042505 (2009). 